\title{
MATHEMATICAL AND PHYSICAL ASPECTS OF THE INITIAL VALUE PROBLEM FOR A NONLOCAL MODEL OF HEAT PROPAGATION WITH FINITE SPEED
}

Abstract. Theories of heat predicting a finite speed of propagation of thermal signals have come into existence during the last 50 years. It is worth emphasizing that in contrast to the classical heat theory, these nonclassical theories involve a hyperbolic type heat equation and are based on experiments exhibiting the actual occurrence of wave-type heat transport (so called second sound). This paper presents a new system of equations describing a nonlocal model of heat propagation with finite speed in the three-dimensional space based on Gurtin and Pipkin's approach. We are interested in the physical and mathematical aspects of this new system of equations. First, using the modified Cagniard-de Hoop method we construct a fundamental solution to this system of equations. Next basing on this fundamental solution, we obtain explicit formulae for the solution of the Cauchy problem to this system. Applying the methods of Sobolev space theory, we get an $L^{p}-L^{q}$ time decay estimate for the solution of the Cauchy problem. For a special form of the source we perform analytical and numerical calculations of the distribution of the temperature for the nonlocal model of heat with finite speed. Some features of the propagation of heat for the nonlocal model are illustrated in a figure together with the comparison of the solution of this model with the solution of the classical heat equation.

1. Introduction. Before we start to describe the nonlocal model of heat propagation at finite speed introduced by Gurtin and Pipkin (cf. [22]), we

2010 Mathematics Subject Classification: Primary 35L40; Secondary 35E05, 35E15, 80A8. Key words and phrases: nonlocal model of heat propagation with finite speed, fundamental solution, modiffied Cagniard-de Hoop's method, Cauchy problem, Sobolev space, $L^{p}-L^{q}$ time decay estimate. 
will describe some theories starting from the classical theory which lead to hyperbolic equations for heat propagation.

The classical theory of heat conduction predicts that if a heat conducting material is subjected to a thermal disturbance, the effects of the disturbance will be felt instantaneously at distances infinitely far from its source. This does not accord with the physical point of view, particularly in problems concerned with sudden heat inputs.

This shortcoming of the theory stems from the fact that the heat transport equation is a parabolic-type partial differential equation that allows an infinite speed of thermal signals. During the last 50 years nonclassical theories free from this drawback have been formulated (cf. [10]).

These new theories make use of a modified version of the classical Fourier law of heat conduction and consequently involve hyperbolic-type heat transport equations admitting finite speeds of thermal signals. According to these theories, heat propagation is viewed as a wave phenomenon rather than a diffusion phenomenon. A wavelike thermal disturbance is referred to as second sound - the first sound being the usual sound wave - and nonclassical theories predicting the occurrence of such disturbances are known as theories with finite wave speeds or theories with second sound. These theories are motivated by experiments exhibiting the actual occurrence of second sound at low temperatures and for small intervals of time.

One of the aims of this paper is to present a review of the equations related to the generalized heat equation. In order to make the exposition fairly complete a brief account of the theory of heat conduction with second sound as well as a nonlocal model of heat propagation with finite speed introduced by Gurtin and Pipkin are presented (cf. [22]).

The main aim of this paper is the investigation of the mathematical and physical properties of the solution of the Cauchy problem to the system of equations describing this nonlocal model. Rouhly speaking, we will prove an $L^{p}-L^{q}$ time decay estimate for the solution of the Cauchy problem for the nonlocal Gurtin and Pipkin model of heat propagation in a rigid heat conductor.

Our paper is organized as follows:

In Section 2, we present an account of the literature relating to experimental and theoretical aspects of the theory of heat conduction with second sound and to the nonlocal Gurtin and Pipkin model, the statement of the problem and the Main Theorem.

In Section 3 we present basic notation and formulae.

Section 4 is devoted to the construction of a fundamental solution to the nonlocal system of equations describing heat propagation with finite speed. The construction is based on the modified Cagniard-de Hoop method. 
In Section 5 we prove an $L^{\infty}-L^{1}$ time decay estimate for the solution of the Cauchy problem for the system given by Gurtin and Pipkin.

In Section 6 we obtain an $L^{2}-L^{2}$ time decay estimate for this solution.

In Section 7 we prove an $L^{p}-L^{q}$ time decay estimate for the solution of the Cauchy problem for the nonlocal model of heat transport.

Section 8 is devoted to the mathematical and physical interpretation of the solution to the nonlocal model of heat propagation with finite speed and its comparison with the solution of the classical heat equation.

In Section 9 we present numerical calculations of the distribution of the temperature for the nonlocal model of heat propagation with a special source, and compare it with the solution of the classical heat equation.

2. Statement of the problem. Main theorem. The classical Fourier law relates the heat flux vector $q$ to the temperature gradient $\nabla T$ through the equation

$$
q=-k \nabla T
$$

where $k>0$ is the thermal conductivity of the material. The conventional theory of heat conduction is based on this equation.

Equation 2.1 leads to the parabolic-type classical heat transport equation

$$
\varrho c \frac{\partial T}{\partial t}=k \nabla^{2} T
$$

where $\varrho>0$ is the mass density, $c>0$ is the specific heat capacity and $t$ is time. Equations (2.1) and $(2.2)$ hold for a homogeneous and isotropic material in the context of the linear theory.

A direct consequence of the form of equation 2.2 is that if the material is subjected to a thermal disturbance, the effects of the disturbance will be felt instantaneously at infinitely far distances. This amounts to saying that thermal signals propagate with infinite speed, which is not in accordance with physical intuition.

Efforts to eliminate the paradox of infinite heat propagation speed have been made for over 140 years $([1],[2],[6],[11$, [13], [14], [16], [23], [27], [28], [32, 33], 34], [55], [56]). Maxwell was the first to postulate in 1867 (cf. [39]) the occurrence of wave-type heat flow, now called second sound, while developing a kinetic theory of gases, and suggested a modification of the Fourier law.

In 1948 and 1958, 1961 some studies from a theoretical point of view were conducted (among others) by Cattaneo (cf. [8], [9]) and Vernotte (cf. [52, [53], 54]), where the existence of second sound was discovered. 
This generalized Fourier law for a homogeneous and isotropic material is given by the equation

$$
\tau \frac{\partial q}{\partial t}+q=-k \nabla T
$$

where $\tau \geq 0$ is a constant. The corresponding heat transport equation is

$$
\varrho c \tau \frac{\partial^{2} T}{\partial t^{2}}+c \frac{\partial T}{\partial t}=k \nabla^{2} T .
$$

This equation is of hyperbolic type and predicts a finite speed $v_{T}=(k / \varrho c \tau)^{1 / 2}$ for heat propagation provided $\tau>0$. The theory of heat conduction based on (2.4) is thus free from the unpleasant paradox of infinite heat propagation speed.

The constant $\tau$ appearing in (2.3) and (2.4) has a definite physical interpretation. It represents the time lag needed to establish the steady state of heat conduction in an element of volume when a temperature gradient is suddenly imposed on that element. This time lag is referred to as the thermal relaxation time (44, [37, [38], [40, 41], 44], 45], [47], [50]).

Equation (2.4) was investigated by Kaliski in 1965 (cf. [29], [30]), Luikov in 1965, 1966 (cf. [35], 36]), Lambermont and Lebon in 1973 (cf. 31]) and Nowacki (cf. [42]).

Gawinecki (cf. [17], [18], [19]) constructed a fundamental solution to 2.4 using a different method from the other ones, based on Hörmander's theorem. Next basing on that fundamental solution he proved an $L^{p}-L^{q}$ time decay estimate for the solution of the Cauchy problem for (2.4). Next, he proved the corresponding global existence theorem for the Cauchy problem in $\mathbb{R}^{3}$ for the nonlinear hyperbolic heat equation (cf. [19]).

We consider a new system of equations describing a nonlocal model of heat propagation with finite speed in the three-dimensional space based on Gurtin and Pipkin's approach. Before describing the main results of the paper we will describe Gurtin and Pipkin's theory.

In 1968 Gurtin and Pipkin [22] formulated a general nonlinear theory of heat conduction that admits second sound, for materials with memory. Their theory was an application of the mathematical method then in use in continuum mechanics and thermodynamics. They showed that their theory differs from other theories of heat conduction in that the heat-flux, like entropy, was determined by the functional of free energy.

Their method required characterizing the domain space of functionals and they chose a weighted $L_{h}^{2}$ space (cf. [12]). It is the space of functions of $s$ whose squares are integrable over $[0, \infty)$ against a decaying positive weight $h(s)$, that is, $s^{2} h(s)$ is integrable.

The Riesz representation theorem then implies that linearized flux laws may be represented by the integral from 0 to $\infty$ of the temperature gradient 
(cf. [22, formulae (1.9)]), and application of Schwarz's inequality then shows that $Q(\cdot) / h(\cdot)$ must be square integrable against $h(s)$. Many other choices for the allowed domain of the free-energy functional are possible and each leads to different laws for heat conduction (cf. 44]).

At the end of the analysis carried out by Gurtin and Pipkin [22, after linearization, they obtained the constitutive equations of the form

$$
\begin{aligned}
& \partial_{t} e+\operatorname{div} q=W(t, x), \\
& e(t, x)=c \theta(t, x)-\int_{0}^{\infty} b(\eta) \theta(t-\eta, x) d \eta, \\
& q(t, x)=-\int_{0}^{\infty} a(\eta) \operatorname{grad} \theta(t-\eta, x) d \eta,
\end{aligned}
$$

where $e=e(t, x)$ is the internal energy, $x \in \mathbb{R}^{3}, q=q(t, x)$ is the heat flux, $\theta=\theta(t, x)>0$ is the absolute temperature, $W=W(t, x)$ is the heat supplied to the body by the external world, $a(\eta)$ is the heat-flux relaxation function, $b(\eta)$ is the energy relaxation function, and $c$ is the coefficient of specific heat.

Equation (2.5) is the equation of energy, while (2.6), (2.7) are the constitutive relations (cf. [22]) with $b(\infty)=0$ and $a(\infty)=0$.

In our paper we consider the following Cauchy problem for the system of equations of the nonlocal theory of heat propagation:

$$
\begin{aligned}
& \partial_{t} e+q_{i, i}=W(t, x), \\
& \partial_{t} q_{k}(t, x)=-a(0) \partial_{k} \theta(t, x)-\int_{0}^{\infty} a^{\prime}(\eta) \operatorname{grad} \theta(t-\eta, x) d \eta, \\
& \partial_{t} e(t, x)=c \partial_{t} \theta(t, x)+b(0) \theta(t, x)+\int_{0}^{\infty} b^{\prime}(\eta) \theta(t-\eta, x) d \eta,
\end{aligned}
$$

with initial conditions

$$
e(0)=e_{0}, \quad q(0)=q_{0}, \quad \theta(0)=\theta_{0}
$$

and the additional condition

$$
\partial_{k} q_{0}=0 .
$$

Equations 2.8)-(2.10) can be written (cf. [22]) as the following integraldifferential equation:

$$
c \partial_{t} \theta(t, x)+\partial_{t} \int_{0}^{t} b(\eta) \theta(t-\eta, x) d \eta-\Delta \int_{0}^{t} a(\eta) \theta(t-\eta, x) d \eta=W(t, x) .
$$


Equation 2.13) describes the propagation of heat with finite speed $U$ equal to

$$
U=\sqrt{a(0) / c}
$$

Under the assumption that the Cauchy data $\theta_{0}, q_{0}, e_{0}$ are smooth enough the solution of the Cauchy problem for the temperature $\theta(t, x)$ can be written as follows:

$$
\theta(t, x)=c \mathbb{E}(t, x-\cdot) *_{3} \theta_{0}(\cdot)
$$

and additionally for the heat flux $q(t, x)$ and internal energy $e(x, t)$ as below:

$$
\begin{aligned}
q_{k}(t, x) & =-a *_{t} \partial_{k}\left(c \mathbb{E}(t, a-x) *_{3} \theta_{0}(\cdot)\right), \\
e(t, x) & =e_{0}+c^{2} \mathbb{E}(t, x-\cdot) *_{3} \theta_{0}(\cdot)+b *_{t}\left(c \mathbb{E}(t, x-\cdot) *_{3} \theta_{0}(\cdot)\right)
\end{aligned}
$$

where $\mathbb{E}(t, x)$ is the fundamental solution of equation 2.13) and $*_{t}$ denotes convolution with respect to $t$, while $*_{3}$ denotes convolution with respect to $x \in \mathbb{R}^{3}$.

The aim of our paper is to prove an $L^{p}-L^{q}$ time decay estimate for the solution of the Cauchy problem for temperature. Now, we formulate our main results:

Theorem 2.1 (Main Theorem, $L^{p}-L^{q}$ time decay estimate). Let $1<p \leq$ $q<\infty, 1 / p+1 / q=1$ and let $s \in \mathbb{N}, s \geq[3(1-2 / q)]+1$. If $\left(\theta_{0}, D \theta_{0}\right) \in$ $W^{s, p}\left(\mathbb{R}^{3}\right)$ then there exists a constant $C>0$ such that for $t>0$ the solution given by 2.14 satisfies the following estimate:

$$
\|(\theta, D \theta)\|_{L^{q}\left(\mathbb{R}^{3}\right)} \leq C(1+t)^{-\frac{3}{2}(1-2 / q)}\left\|\left(\theta_{0}, D \theta_{0}\right)\right\|_{W^{s, p}\left(\mathbb{R}^{3}\right)}
$$

where $D \theta=\left(\partial_{1} \theta, \partial_{2} \theta, \partial_{3} \theta\right)^{T}, D \theta_{0}=\left(\partial_{1} \theta_{0}, \partial_{2} \theta_{0}, \partial_{3} \theta_{0}\right)^{T}$.

$L^{p}-L^{q}$ time decay estimates for solutions of linear evolution equations can be written in the general forms

$$
\|V(t)\|_{L^{q}\left(\mathbb{R}^{n}\right)} \leq c(1+t)^{-d}\left\|V^{0}\right\|_{W^{N_{p}, p}\left(\mathbb{R}^{n}\right)}
$$

where $2 \leq q<\infty, 1 / p+1 / q=1, c, d>0, N_{p}>n(1-2 / q)$, and $n$ is the space dimension.

1. For the wave equation in three dimensions $(n=3)$ we have $d=1-2 / q$.

2. For the classical heat equation in three dimensions we have $d=$ $\frac{3}{2}(1-2 / q)$.

In our model, i.e. for the nonlocal model of heat propagation with finite speed (cf. Main Theorem 2.1. 22.17) we prove that $d=\frac{3}{2}(1-2 / q$ ).

So, the time decay for the nonlocal model of heat propagation is similar to the time decay of the parabolic heat equation but strongly depends on the relaxation functions $a(t)$ and $b(t)$ (cf. formulae (4.9), (4.10)). Hence, our nonlocal model is more general than the classical one describing the 
propagation of heat and the model describing the propagation of heat by the hyperbolic partial differential equation (cf. [19]).

Additionally, roughly speaking the nonlocal model of heat propagation with finite speed is essentially different from the classical description of propagation of heat (cf. Fig. 4) from the following points of view:

$1^{\circ}$ the wave front exists in this description (cf. Fig. 5),

$2^{\circ}$ the distribution of the temperature exists in a neighbourhood of the wave front (cf. Fig. 5).

Before starting the proof of the main theorem we introduce some notation and some facts on Sobolev spaces.

3. Basic notation and formulae. Let us assume that $0<s \leq \infty$, $1 \leq p<\infty$. We denote by $W^{s, p}\left(\mathbb{R}^{n}\right)$ the usual Sobolev space, i.e.

$$
\begin{aligned}
& W^{s, p}\left(\mathbb{R}^{n}\right)=\left\{u: D^{\alpha} u \in L^{p}\left(\mathbb{R}^{n}\right) \text { for }|\alpha| \leq s\right. \\
& \left.\qquad\|u\|_{W^{s, p}\left(\mathbb{R}^{n}\right)}=\left(\sum_{|\alpha| \leq s}\left\|D^{\alpha} u\right\|_{L^{p}\left(\mathbb{R}^{n}\right)}^{p}\right)^{1 / p}<\infty\right\} .
\end{aligned}
$$

Write

$$
W^{s, 2}\left(\mathbb{R}^{n}\right)=H^{s}\left(\mathbb{R}^{n}\right)
$$

Let

$$
S\left(\mathbb{R}^{n}\right)=\left\{u \in C^{\infty}\left(\mathbb{R}^{n}\right): \forall \alpha, \beta \sup _{x \in \mathbb{R}^{n}}\left|x^{\beta} \partial^{\alpha} u(x)\right|<\infty\right\}
$$

denote the space of infinitely differentiable functions, rapidly decreasing as $|x| \rightarrow \infty$.

By $S^{\prime}\left(\mathbb{R}^{n}\right)$ we will denote the space of tempered distributions. Below, we recall the definition of the Fourier transformation.

Definition 3.1. The Fourier transform of a function $u \in S\left(\mathbb{R}^{n}\right)$ is the function denoted by $\mathcal{F} u$ or $\hat{u}$ defined by the formula

$$
\mathcal{F} u(\xi)=\hat{u}(\xi)=\frac{1}{(2 \pi)^{n / 2}} \int_{\mathbb{R}^{n}} u(x) e^{-i x \xi} d x .
$$

The inverse Fourier transform of a function $\hat{u} \in S\left(\mathbb{R}^{n}\right)$ is given by

$$
\mathcal{F}^{-1} \hat{u}(x)=u(x)=\frac{1}{(2 \pi)^{n / 2}} \int_{\mathbb{R}^{n}} \hat{u}(\xi) e^{i x \xi} d \xi .
$$

The Fourier transform of a distribution $u \in S^{\prime}\left(\mathbb{R}^{n}\right)$ is the distribution $\hat{u} \in$ $S^{\prime}\left(\mathbb{R}^{n}\right)$ defined as follows:

$$
\langle\hat{u}, \varphi\rangle=\langle u, \hat{\varphi}\rangle \quad \text { for } \varphi \in S\left(\mathbb{R}^{n}\right) .
$$

The Fourier transform is an automorphism of $S$ and of $S^{\prime}$ (cf. [48]). 
Definition 3.2. The convolution of $u, v \in S\left(\mathbb{R}^{n}\right)$ is the function $u *_{n} v$ defined by

$$
\left(u *_{n} v\right)(x)=\int_{\mathbb{R}^{n}} u(x-y) v(y) d y .
$$

The convolution of a distribution $u \in S^{\prime}\left(\mathbb{R}^{n}\right)$ with a function $\varphi \in S\left(\mathbb{R}^{n}\right)$ is the function defined by

$$
\left(u *_{n} \varphi\right)(x)=\langle u, \overline{\varphi(x-\cdot)}\rangle .
$$

Now, we will introduce some notations and properties concerning Besov and Bessel spaces (cf. [3], 25], [49], [51]). Let $\varphi$ be an arbitrary nonnegative function satisfying the conditions

$$
\begin{gathered}
\varphi \in C_{0}^{\infty}\left(\mathbb{R}^{n}\right), \quad \operatorname{supp} \varphi \subset\left\{x \in \mathbb{R}^{n}: 1 / 2 \leq|x| \leq 2\right\}, \\
\varphi(x)>0 \quad \text { for } 1 / 2<|x|<2, \quad \sum_{k=-\infty}^{\infty} \varphi\left(2^{-k} x\right)=1 \quad \text { for } x \neq 0 .
\end{gathered}
$$

For the given $\varphi$ we define the functions $\varphi_{k}, k \in \mathbb{Z}$, and $\psi$ by the formulae

$$
\mathcal{F} \varphi_{k}(\xi)=\varphi\left(2^{-k} \xi\right), \quad \mathcal{F} \psi(\xi)=1-\sum_{k=1}^{\infty} \mathcal{F} \varphi\left(2^{-k} \xi\right) .
$$

Definition 3.3. Let $1 \leq p \leq q \leq \infty$ and $s>0$. The Bessel space is

$$
H^{s, p}\left(\mathbb{R}^{n}\right)=\left\{u \in S^{\prime}\left(\mathbb{R}^{n}\right):\|u\|_{H^{s, p}\left(\mathbb{R}^{n}\right)}<\infty\right\}
$$

where

$$
\|u\|_{H^{s, p}\left(\mathbb{R}^{n}\right)}=\left\|\mathcal{F}^{-1}\left(1+|\xi|^{2}\right)^{s / 2} \mathcal{F} u\right\|_{L^{p}\left(\mathbb{R}^{n}\right)} .
$$

The Besov space is

$$
B_{p, q}^{s}\left(\mathbb{R}^{n}\right)=\left\{u \in S^{\prime}\left(\mathbb{R}^{n}\right):\|u\|_{B_{p, q}^{s}\left(\mathbb{R}^{n}\right)}<\infty\right\}
$$

where

$$
\|u\|_{B_{p, q}^{s}\left(\mathbb{R}^{n}\right)}=\left(\left\|\psi *_{n} u\right\|_{L^{p}\left(\mathbb{R}^{n}\right)}^{q}+\sum_{k=1}^{\infty}\left(2^{s k}\left\|\varphi_{k} *_{n} u\right\|_{L^{p}\left(\mathbb{R}^{n}\right)}\right)^{q}\right)^{1 / q} .
$$

Below, we give some theorems which are necessary in order to get an $L^{p}-L^{q}$ time decay estimate (cf. [51]).

Theorem 3.1. If $m \in \mathbb{N}_{0}, 1<p<\infty$, then

$$
W^{m, 2}\left(\mathbb{R}^{n}\right)=H^{m, 2}\left(\mathbb{R}^{n}\right)=B_{22}^{m}\left(\mathbb{R}^{n}\right), \quad W^{m, p}\left(\mathbb{R}^{n}\right)=H^{m, p}\left(\mathbb{R}^{n}\right) .
$$

Theorem 3.2. If $m \in \mathbb{N}_{0}, 1 \leq p<\infty$, then

$$
B_{p, 1}^{m}\left(\mathbb{R}^{n}\right) \hookrightarrow W^{m, p}\left(\mathbb{R}^{n}\right) .
$$

Theorem 3.3. If $s \geq 0,1<p<\infty, 1 \leq q \leq \infty$, $\varepsilon>0$, then $H^{s+\varepsilon, p}\left(\mathbb{R}^{n}\right) \hookrightarrow B_{p, q}^{s}\left(\mathbb{R}^{n}\right)$. 
Theorem 3.4. Let $\theta \in(0,1), p_{0}, p_{1} \in[1, \infty]$ and

$$
\frac{1}{p_{\theta}}=\frac{1-\theta}{p_{0}}+\frac{\theta}{p_{1}} \text {. }
$$

Then

$$
\left[L^{p_{0}}\left(\mathbb{R}^{n}\right), L^{p_{1}}\left(\mathbb{R}^{n}\right)\right]_{\theta}=L^{p_{\theta}}\left(\mathbb{R}^{n}\right)
$$

where $\left[L^{p_{0}}\left(\mathbb{R}^{n}\right), L^{p_{1}}\left(\mathbb{R}^{n}\right)\right]_{\theta}$ is the interpolation space between $L^{p_{0}}\left(\mathbb{R}^{n}\right)$ and $L^{p_{1}}\left(\mathbb{R}^{n}\right)$.

Theorem 3.5. Let $\theta \in(0,1), p_{0}, q_{0}, p_{1}, q_{1} \in[1, \infty]$ and

$$
\frac{1}{p_{\theta}}=\frac{1-\theta}{p_{0}}+\frac{\theta}{p_{1}}, \quad \frac{1}{q_{\theta}}=\frac{1-\theta}{q_{0}}+\frac{\theta}{q_{1}}, \quad s_{\theta}=(1-\theta) s_{0}+\theta s_{1} .
$$

Then

$$
\left[B_{p_{0}, q_{0}}^{s_{0}}\left(\mathbb{R}^{n}\right), B_{p_{1}, q_{1}}^{s_{1}}\left(\mathbb{R}^{n}\right)\right]_{\theta}=B_{p_{\theta}, q_{\theta}}^{s_{\theta}}\left(\mathbb{R}^{n}\right) .
$$

TheOREm 3.6. Let $\left(X_{A}, X_{B}\right),\left(Y_{A}, Y_{B}\right)$ be a pair of interpolation spaces, and $T$ a linear operator

$$
T: X_{A}+X_{B} \rightarrow Y_{A}+Y_{B} .
$$

If $T$ is a continuous operator in the spaces $X_{A}, X_{B}$ and

$$
\begin{array}{ll}
\left.T\right|_{X_{A}}: X_{A} \rightarrow Y_{A} & \text { with the norm }\|T\|_{A}, \\
\left.T\right|_{X_{B}}: X_{B} \rightarrow Y_{B} & \text { with the norm }\|T\|_{B},
\end{array}
$$

then for $\theta \in(0,1)$ the operator

$$
\left.T\right|_{\left[X_{A}, X_{B}\right]_{\theta}}:\left[X_{A}, X_{B}\right]_{\theta} \rightarrow\left[Y_{A}, Y_{B}\right]_{\theta}
$$

is a continuous operator and its norm satisfies

$$
\|T\|_{\theta} \leq\|T\|_{A}^{1-\theta}\|T\|_{B}^{\theta} .
$$

4. Construction of a fundamental solution for the nonlocal model of heat propagation. In order to prove the main theorem we start with the construction of a fundamental solution for the nonlocal model of heat propagation. We proved (cf. 2.13) that equations (2.8)-2.11) may be written as follows:

$$
\begin{aligned}
c \partial_{t} E(t, x)+\partial_{t} \int_{0}^{t} b(\eta) E(t-\eta, x) d \eta-\Delta \int_{0}^{t} a(\eta) E(t- & \eta, x) d \eta \\
= & W_{0} \delta(t) \delta(x)
\end{aligned}
$$

where $\delta(t), \delta(x)$ denote Dirac distributions (cf. [55]) and $E(t, x)$ expresses that we are looking for the fundamental solution of our problem with source of the form $W(t, x)=W_{0} \delta(t) \delta(x)$. 
Applying the Laplace transformation with respect to $t$ and the Fourier transformation with respect to $x_{1}, x_{2}$ given by formula (3.1) to the system (2.8)-2.12) we get

$$
\left(s(c+\bar{b}(s))+\bar{a}(s)\left(\xi_{1}^{2}+\xi_{2}^{2}\right)\right) \hat{\overline{\mathbb{E}}}-\bar{a}(s) \frac{d^{2} \hat{\overline{\mathbb{E}}}}{d x_{3}^{2}}=\hat{\bar{W}}
$$

where $\bar{a}(s), \bar{b}(s)$ denote the Laplace transforms of the relaxation functions $a(\cdot), b(\cdot)$, and

$$
\hat{\overline{\mathbb{E}}}\left(s, \xi_{1}, \xi_{2}, x_{3}\right) \equiv \int_{\mathbb{R}^{2}} e^{-i\left(\xi_{1} x_{1}+\xi_{2} x_{2}\right)}\left[\int_{0}^{\infty} \mathbb{E}(t, x) e^{-s t} d t\right] d x_{1} d x_{2} .
$$

Moreover $\hat{\bar{W}}$ denotes the Laplace-Fourier transform of the unit source $W(t, x)$ $=W_{0} \delta(t) \delta(x)$ which is given by

$$
\hat{\bar{W}}=W_{0} \delta\left(x_{3}\right)
$$

where

$$
a(\eta)=0, \quad b(\eta)=0 \quad \text { for } \eta<0 .
$$

Solving equation 4.1 we get

$$
\hat{\overline{\mathbb{E}}}\left(s, \xi_{1}, \xi_{2}, x_{3}\right)=\frac{W_{0}}{2 \overline{a(s)} \gamma} e^{-\gamma\left|x_{3}\right|}
$$

where

$$
\begin{aligned}
\gamma & =\sqrt{\xi_{1}^{2}+\xi_{2}^{2}+\bar{r}^{2}}, \quad \operatorname{Re} \gamma>0, \\
\bar{r}(s) & =\sqrt{\frac{s(c+\bar{b})}{\bar{a}}} .
\end{aligned}
$$

In order to get the fundamental solution $E(t, x)$ in space-time we could use the formula for the inverse Fourier and Laplace transformation. It leads to a singular integral, from which it is difficult to obtain the solution in explicit form. We can see this from the following formula for $\mathbb{E}(t, x)$ which is given by a triple integral:

$$
\begin{aligned}
\mathbb{E}(t, x) & =\mathcal{L}^{-1} \mathcal{F}_{\xi_{1}, \xi_{2}}^{-1} \hat{\overline{\mathbb{E}}}\left(s, \xi_{1}, \xi_{2}, x_{3}\right) \\
& =\frac{1}{2 \pi i} \int_{c-i \infty}^{c+i \infty}\left(\frac{1}{4 \pi^{2}} \int_{\mathbb{R}^{2}} \frac{W_{0} e^{-\sqrt{\xi_{1}^{2}+\xi_{2}^{2}+\bar{r}^{2}}\left|x_{3}\right|}}{2 \hat{a}(s) \sqrt{\xi_{1}^{2}+\xi_{2}^{2}+\bar{r}^{2}}} e^{i\left(x_{1} \xi_{1}+x_{2} \xi_{2}\right)} d \xi_{1} d \xi_{2}\right) e^{s t} d s .
\end{aligned}
$$

In order to circumvent these dificulties we will apply the Cagniard method (cf. [7]) and its modification made by de Hoop (cf. [24]). The Cagniard-de Hoop method is based on integration along a specially chosen contour $\Gamma$ on the complex plane of $\omega$. The integration contour $\Gamma$ is described parametrically by formula 4.4 and presented in Fig. 1 


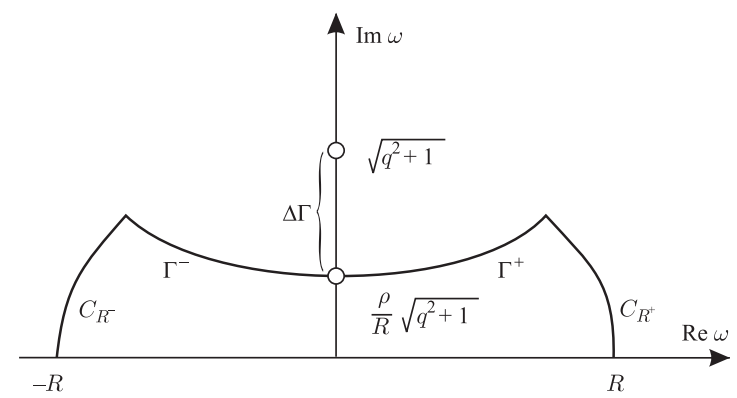

Fig. 1

Using the modified de Hoop transformation (cf. [24]) in the form $\xi_{1}=\left(\omega \frac{x_{1}}{\rho}+q \frac{x_{2}}{\rho}\right) \bar{r}, \quad \xi_{2}=\left(\omega \frac{x_{2}}{\rho}-q \frac{x_{1}}{\rho}\right) \bar{r} \quad$ where $\quad \rho=\sqrt{x_{1}^{2}+x_{2}^{2}}$,

we get

$$
\overline{\mathbb{E}}\left(s, \rho, x_{3}\right)=\frac{W_{0}}{4 \pi^{2}} \int_{-\infty}^{\infty} d q \int_{-\infty}^{\infty} \frac{\bar{r}}{2 \bar{a}} \frac{e^{\left(i \omega \rho-\left|x_{3}\right| \sqrt{\omega^{2}+q^{2}+1}\right) \bar{r}}}{\sqrt{\omega^{2}+q^{2}+1}} d \omega .
$$

Using the modified Cagniard method (cf. [7], [24]) we replace the integral with respect to $\omega$ (after a suitable transformation) by the integral over a suitably chosen contour $\Gamma$ (cf. Fig. 1):

$$
\Gamma:\left|x_{3}\right| \sqrt{\omega^{2}+q^{2}+1}-i \omega \rho=T, \quad T \geq 0 .
$$

From 4.4), we get

$$
\omega^{ \pm}=i \frac{T \rho}{|x|^{2}} \pm \frac{\left|x_{3}\right|}{|x|^{2}} \sqrt{T^{2}-|x|^{2}\left(q^{2}+1\right)}
$$

where $|x|=\sqrt{x_{1}^{2}+x_{2}^{2}+x_{3}^{2}}=\sqrt{\rho^{2}+x_{3}^{2}}, T>|x| \sqrt{q^{2}+1}$.

We find that

$$
\begin{aligned}
& \int_{-R}^{+R} f(\omega) d \omega+\int_{C_{R^{+}}} f(\omega) d \omega+\int_{C_{R^{-}}} f(\omega) d \omega+\int_{\Delta \Gamma} f(\omega) d \omega \\
& \quad+\int_{\Gamma^{+}} f\left(\omega^{+}(T)\right) d \omega^{+}(T)+\int_{\Gamma^{-}} f\left(\omega^{-}(T)\right) d \omega^{-}(T)=2 \pi i \sum_{\operatorname{res} \omega_{j}} f\left(\omega_{j}\right) .
\end{aligned}
$$

The integrals over the contours $C_{R^{+}}$and $C_{R^{-}}$vanish, so in view of the Jordan Lemma, after some calculations we get

$$
\overline{\mathbb{E}}(s, x)=\left.\frac{W_{0}}{4 \pi^{2}} \int_{-\infty}^{\infty} d q \int_{|x| \sqrt{q^{2}+1}}^{\infty} \frac{\bar{r}}{2 \bar{a}} \frac{e^{\left(i \omega \rho-\left|x_{3}\right| \sqrt{\omega^{2}+q^{2}+1}\right) \bar{r}}}{\sqrt{\omega^{2}+q^{2}+1}} \frac{\partial \omega}{\partial T}\right|_{\omega=\omega^{-}} ^{\omega=\omega^{+}} d T
$$


and next

$$
\overline{\mathbb{E}}(s, x)=\frac{W_{0}}{4 \pi^{2}} \int_{-\infty}^{\infty} \bar{r} d q \int_{|x| \sqrt{q^{2}+1}}^{\infty} \frac{e^{-\bar{r} T}}{\bar{a} \sqrt{T^{2}-|x|^{2}\left(q^{2}+1\right)}} d T .
$$

Since the integrand has no singularities inside the integral contour, we get

$$
\overline{\mathbb{E}}(s, x)=\frac{W_{0}}{4 \pi} \int_{|x|}^{\infty} \frac{\bar{r}}{\bar{a}|x|} e^{-\bar{r} T} d T=\frac{e^{-\bar{r}|x|}}{4 \pi \bar{a}|x|} .
$$

So, we have

$$
\overline{\mathbb{E}}(t, x)=\frac{W_{0}}{4 \pi} \mathcal{L}^{-1}\left[\frac{e^{-\bar{r}|x|}}{\bar{a}|x|}\right]
$$

where $\mathcal{L}^{-1}$ denotes the inverse Laplace transformation.

Putting in 4.5) $\bar{a}(s)=a_{0}=$ const, $\bar{b}(s)=0\left(a(t)=a_{0} \delta(t), b(t)=0\right.$ and $\left.W_{0}=1\right)$ we get

$$
\mathbb{E}(t, x)=\frac{1}{4 \pi a_{0}|x|} \mathcal{L}^{-1}\left[e^{-\sqrt{|x|^{2} s c / a_{0}}}\right] .
$$

From this transformation we deduce (cf. [15])

$$
\mathbb{E}(t, x)=\frac{e^{-\frac{|x|^{2}}{4 D^{2} t}}}{D a_{0}(\sqrt{4 \pi t})^{3}}
$$

where $D=\sqrt{a_{0} / c}, a_{0}$ denotes the heat coefficient, and $D$ is the heat diffusion coefficient. Formula 4.8 gives the fundamental solution of the classical heat equation.

In order to obtain the explicit representation for the temperature given by formula (4.6) we assume that the relaxation functions are given in Maxwell form (cf. [22]):

$$
\begin{aligned}
& a(t)=a_{0} \frac{1}{\tau} e^{-t / \tau}, \\
& b(t)=b_{0} e^{-t / \tau},
\end{aligned}
$$

where $a_{0}, b_{0}, \tau$ denote the amplitudes and the relaxation time, respectively. From 4.9 and 4.10 we obtain

$$
\bar{a}(s)=a_{0} \frac{1}{\tau} \frac{1}{s+1 / \tau}, \quad \bar{b}(s)=b_{0} \frac{1}{s+1 / \tau},
$$

Hence

$$
\mathbb{E}(t, x)=\frac{W_{0}}{4 \pi a_{0}} \mathcal{L}^{-1}\left[(\tau s+1) \frac{e^{-\frac{|x|}{U} \sqrt{s\left(s+1 / \tau+b_{0} / c\right)}}}{|x|}\right]
$$


where $U=\sqrt{a_{0} / c \tau}$. Finally, after some calculations, we have

$$
\mathbb{E}(t, x)=\frac{W_{0}}{4 \pi a_{0}}\left[\left(1+\tau \frac{d}{d t}\right) \mathbb{E}_{3}(t, x)\right]
$$

where

$$
\begin{aligned}
\mathbb{E}_{3}(t, x)= & e^{-\frac{1}{2}\left(1 / \tau+b_{0} / c\right) t} \frac{1}{|x|}\left(\delta\left(t-\frac{|x|}{U}\right)+\frac{\frac{1}{2}\left(\frac{1}{\tau}+\frac{b_{0}}{c}\right) \frac{|x|}{U}}{\sqrt{t^{2}-|x|^{2} / U^{2}}}\right. \\
& \left.\cdot I_{1}\left(\frac{1}{2}\left(\frac{1}{\tau}+\frac{b_{0}}{c}\right) \sqrt{t^{2}-\frac{|x|^{2}}{U^{2}}}\right) H\left(t-\frac{|x|}{U}\right)\right) .
\end{aligned}
$$

In 4.12), $I_{1}(\cdot)$ denotes the modified Bessel function of the first order (cf. [15],

$$
I_{\nu}(z)=\left(\frac{z}{2}\right)^{\nu} \sum_{k=0}^{\infty} \frac{(z / 2)^{2 k}}{k ! \Gamma(\nu+k+1)},
$$

$H(\cdot)$ denotes the Heaviside function, $\delta(t-|x| / U)$ is the Dirac distribution concentrated on the sphere with radius $|x|=U t$ (cf. [5], [54]) and describing the wave front moving with velocity $U$.

5. $L^{\infty}-L^{1}$ time decay estimates for the solution of the Cauchy problem in nonlocal theory. We showed (cf. 2.14)-(2.16) ) that the solution of the Cauchy problem for the system of equations of the nonlocal theory of heat propagation,

$$
\begin{aligned}
& \partial_{t} e+q_{i, i}=0 \\
& \partial_{t} q_{k}(t, x)=-a(0) \partial_{k} \theta(t, x)-\int_{0}^{\infty} a^{\prime}(\eta) \operatorname{grad} \theta(t-\eta, x) d \eta, \\
& \partial_{t} e(t, x)=c \partial_{t} \theta(t, x)+b(0) \theta(t, x)+\int_{0}^{\infty} b^{\prime}(\eta) \theta(t-\eta, x) d \eta,
\end{aligned}
$$

with initial conditions

$$
e(0)=e_{0}, \quad q(0)=q_{0}, \quad \theta(0)=\theta_{0}
$$

and with the additional condition

$$
\partial_{k} q_{0}=0
$$

can be represented in the form

$$
\theta(t, x)=c \mathbb{E}(t, x-\cdot) *_{3} \theta_{0}(\cdot)
$$


and

$$
\begin{aligned}
q_{k}(t, x) & =-a *_{t} \partial_{k}\left(c \mathbb{E}(t, x-\cdot) *_{3} \theta_{0}(\cdot)\right), \\
e(t, x) & =e_{0}+c^{2} \mathbb{E}(t, x-\cdot) *_{3} \theta_{0}(\cdot)+b *_{t}\left(c \mathbb{E}(t, x-\cdot) *_{3} \theta_{0}(\cdot)\right),
\end{aligned}
$$

where $\mathbb{E}(t, x)$ is the fundamental solution given by 4.11 and $*_{t}$ denotes convolution with respect to $t$.

Now, we shall prove the following theorem.

TheOrem $5.1\left(L^{\infty}-L^{1}\right.$ time decay estimate). If

$$
\left(\theta_{0}, D \theta_{0}\right) \in W^{3,1}\left(\mathbb{R}^{3}\right)
$$

then the solution $\theta$ given by formula (5.6) satisfies

$$
\|(\theta(t, \cdot), D \theta(t, \cdot))\|_{L^{\infty}\left(\mathbb{R}^{3}\right)} \leq C(1+t)^{-3 / 2}\left\|\left(\theta_{0}, D \theta_{0}\right)\right\|_{W^{3,1}\left(\mathbb{R}^{3}\right)}
$$

for $t>0$, where $C$ is a constant independent of $\theta_{0}$ and $t$.

Sketch of proof. Basing on formula 4.11 we can write the expression (5.6) as follows (without loss of generality we assume that $c=1$ ):

$$
\theta(t, x)=\mathbb{E}_{3}(t, x-\cdot) *_{3} \theta_{0}(\cdot)+\tau \partial_{t} \mathbb{E}_{3}(t, x-\cdot) *_{3} \theta_{0}(\cdot)
$$

where

$$
\begin{aligned}
\mathbb{E}_{3}(t, x)= & \frac{U}{4 \pi a_{0}|x|} e^{-m t} \delta(U t-|x|) \\
& +\frac{m e^{-m t}}{4 \pi a_{0}} \frac{I_{1}\left(\frac{m}{U} \sqrt{U^{2} t^{2}-|x|}\right)}{\sqrt{U^{2} t^{2}-|x|^{2}}} \cdot H(U t-|x|)
\end{aligned}
$$

where $m=\frac{1}{2}\left(1 / \tau+b_{0} / c\right)$, that is, $m=U^{2}\left(c+b_{0} \tau\right) / 2 a_{0}$.

Differentiating (5.7) with respect to $x_{l}(l=1,2,3)$ we get

$$
\partial_{l} \theta(t, x)=\mathbb{E}_{3}(t, x-\cdot) *_{3} \partial_{l} \theta_{0}(\cdot)+\tau \partial_{t} \mathbb{E}_{3}(t, x-\cdot) *_{3} \partial_{l} \theta_{0}(\cdot) .
$$

The relations 5.7 and $(5.8)$ can be written in vector form as follows:

$$
\left(\begin{array}{c}
\theta \\
D \theta
\end{array}\right)=\mathbb{B}(t, \cdot) *_{3}\left(\begin{array}{c}
\theta_{0} \\
D \theta_{0}
\end{array}\right)
$$

where

$$
D \theta=\left(\partial_{1} \theta, \partial_{2} \theta, \partial_{3} \theta\right)^{T}, \quad D \theta_{0}=\left(\partial_{1} \theta_{0}, \partial_{2} \theta_{0}, \partial_{3} \theta_{0}\right)^{T}
$$

and $\mathbb{B}(t, x)$ is the $4 \times 4$ matrix

$$
\mathbb{B}(t, x)=\left(\mathbb{E}_{3}(t, x)+\tau \partial_{t} \mathbb{E}_{3}(t, x)\right) I_{4}
$$

where $I_{4}$ is the $4 \times 4$ identity matrix. 
Using the representation of the modified Bessel function (cf. formula (4.13) ) we can write the fundamental solution $\mathbb{E}$ as follows $\left({ }^{1}\right)$.

$$
\begin{aligned}
\mathbb{E}_{3}(t, x)= & \frac{1}{4 \pi a_{0}|x|} e^{-m t \delta} \delta(U t-|x|) \\
& +\frac{1}{2 a_{0} \pi} e^{-m t} \sum_{k=0}^{\infty} \frac{m^{k+2}\left(U^{2} t^{2}-|x|^{2}\right)^{k}}{k !(k+1) !(2 U)^{2 k+2}} H(U t-|x|) .
\end{aligned}
$$

Taking into account $(5.10)$ we get

$$
\begin{aligned}
\mathbb{E}_{3}(t, \cdot) *_{3} f(x-\cdot) & =\frac{1}{4 \pi a_{0} U} t e^{-m t} \int_{|y|=U} f(x+t y) d S_{y} \\
& +\frac{1}{2 a_{0} \pi} \sum_{k=0}^{\infty} \frac{m^{2 k+2} t^{2 k+3} e^{-m t}}{k !(k+1) !(2 U)^{2 k+2}} \int_{|y| \leq U}\left(U^{2}-|y|^{2}\right)^{k} f(x+t y) d y .
\end{aligned}
$$

Estimating the terms $\mathbb{E}_{3}(t, \cdot) *_{3} f(x-\cdot)$ given $\partial_{t} \mathbb{E}_{3}(t, \cdot) *_{3} f(x-\cdot)$ as in [20] and putting all the estimates together, we get

$$
\left\|\mathbb{B}(t, \cdot) *_{3} f\right\|_{L^{\infty}\left(\mathbb{R}^{3}\right)} \leq C(1+t)^{-3 / 2}\|f\|_{W^{3,1}\left(\mathbb{R}^{3}\right)} .
$$

This finishes the proof of Theorem 5.1 .

6. $L^{2}-L^{2}$ time decay estimate for the solution of the Cauchy problem. In order to prove an $L^{2}-L^{2}$ time decay estimate for the solution of the Cauchy problem (5.9) we can write the fundamental solution $\mathbb{E}_{3}(t, x)$ in the form

$$
\mathbb{E}_{3}(t, x)=\frac{e^{-m t}}{a_{0}} R_{1}(t, x)+\frac{m e^{-m t}}{4 \pi a_{0}} R_{2}(t, x)
$$

where

$$
R_{1}(t, x)=\frac{1}{4 \pi U^{2}|x|} \delta\left(t-\frac{|x|}{U}\right)
$$

which is the fundamental solution of the wave operator $P(\partial)=\partial_{t}^{2}-U^{2} \Delta$, and

$$
R_{2}(t, x)=\frac{I_{1}\left(\frac{m}{U} \sqrt{U^{2} t^{2}-|x|^{2}}\right)}{\sqrt{U^{2} t^{2}-|x|^{2}}} H(U t-|x|) .
$$

We can prove the following theorem:

TheOREM 6.1. If $\left(\theta_{0}, D \theta_{0}\right) \in L^{2}\left(\mathbb{R}^{3}\right)$, then there exists a constant $C>0$ such that for $t>0$ the solution given by 2.14 satisfies

$$
\|(\theta(t, \cdot), D \theta(t, \cdot))\|_{L^{2}\left(\mathbb{R}^{3}\right)} \leq C\left\|\left(\theta_{0}, D \theta_{0}\right)\right\|_{L^{2}\left(\mathbb{R}^{3}\right)} .
$$

$\left({ }^{1}\right)$ We change the variables in the convolutions as follows: $z-x=t y, d S_{z}=t^{2} d S_{y}$. 
Proof. In view of (5.6) and 6.1 - 6.3) we can write

$$
\begin{aligned}
\theta(t, x)= & \frac{e^{-m t}}{a_{0}} R_{1}(t, x-\cdot) *_{3} \theta_{0}+\frac{\tau}{a_{0}} \frac{\partial}{\partial t}\left(e^{-m t} R_{1}(t, x-\cdot) *_{3} \theta_{0}\right) \\
& +\frac{m e^{-m t}}{4 \pi a_{0}} R_{2}(t, x-\cdot) *_{3} \theta_{0}+\frac{\tau m}{4 \pi a_{0}} \frac{\partial}{\partial t}\left(e^{-m t} R_{2}(t, x-\cdot) *_{3} \theta_{0}\right),
\end{aligned}
$$

so we get

$$
\begin{aligned}
\theta(t, x)= & \left(\frac{1}{a_{0}}-\frac{\tau m}{a_{0}}\right) e^{-m t} W_{1}(t, x)+\frac{\tau e^{-m t}}{a_{0}} \frac{\partial W_{1}}{\partial t}(t, x) \\
& +\left(\frac{m}{4 \pi a_{0}}-\frac{m^{2} \tau}{4 \pi a_{0}}\right) e^{-m t} W_{2}(t, x)+\frac{\tau m}{4 \pi a_{0}} e^{-m t} \frac{\partial W_{2}(t, x)}{\partial t}
\end{aligned}
$$

where $W_{1}(t, x)=R_{1}(t, x-\cdot) *_{3} \theta_{0}(\cdot), W_{2}(t, x)=R_{2}(t, x-\cdot) *_{3} \theta_{0}(\cdot)$.

The function $W_{1}$ is the solution to the Cauchy problem

$$
\frac{\partial^{2} W_{1}}{\partial t^{2}}-U^{2} \Delta W_{1}=0, \quad W_{1}(0, x)=0, \quad \frac{\partial W_{1}}{\partial t}(0, x)=\theta_{0} .
$$

In order to get estimates for $\left\|W_{1}\right\|_{L^{2}\left(\mathbb{R}^{3}\right)}$ and $\left\|\partial_{t} W_{1}\right\|_{L^{2}\left(\mathbb{R}^{3}\right)}$ we use the following lemma (cf. [26]).

Lemma 6.1. If $a \in L^{\infty}\left(\mathbb{R}^{3}\right)$ and $v \in S\left(\mathbb{R}^{3}\right)$ and $\|a\|_{L^{\infty}\left(\mathbb{R}^{3}\right)}<C$, then

$$
\left\|\mathcal{F}^{-1}(a \hat{v})\right\|_{L^{2}\left(\mathbb{R}^{3}\right)} \leq\|a\|_{L^{\infty}\left(\mathbb{R}^{3}\right)}\|v\|_{L^{2}\left(\mathbb{R}^{3}\right)} .
$$

In view of this fact, we get the following lemma.

Lemma 6.2. Let $W_{1}$ be the solution to the Cauchy problem (6.5). Then for $t \geq 0$,

$$
\left\|\partial_{t} W_{1}\right\|_{L^{2}\left(\mathbb{R}^{3}\right)} \leq C\left\|\theta_{0}\right\|_{L^{2}\left(\mathbb{R}^{3}\right)}, \quad\left\|W_{1}\right\|_{L^{2}\left(\mathbb{R}^{3}\right)} \leq C t\left\|\theta_{0}\right\|_{L^{2}\left(\mathbb{R}^{3}\right)} .
$$

Proof. The Fourier transform of $W_{1}$ is

$$
\hat{W}_{1}=\hat{\theta}_{0} \frac{\sin (U|\xi| t)}{|\xi|}, \quad \partial_{t} \hat{W}_{1}=\hat{\theta}_{0} U \cos (U|\xi| t) .
$$

Putting $a=U \cos (U|\xi| t)$ resp. $a=\frac{\sin (t U|\xi|)}{t U|\xi|}$ we obtain, from Lemma 6.1,

$$
\left\|\partial_{t} W_{1}\right\|_{L^{2}\left(\mathbb{R}^{3}\right)}=\left\|\mathcal{F}^{-1}\left(U \cos (U|\xi| t) \hat{\theta}_{0}\right)\right\|_{L^{2}\left(\mathbb{R}^{3}\right)} \leq C\left\|\theta_{0}\right\|_{L^{2}\left(\mathbb{R}^{3}\right)}
$$

and

$$
\begin{aligned}
\left\|W_{1}\right\|_{L^{2}\left(\mathbb{R}^{3}\right)} & =\left\|\mathcal{F}^{-1}\left(\frac{\sin (t U|\xi|)}{|\xi|} \hat{\theta}_{0}\right)\right\|_{L^{2}\left(\mathbb{R}^{3}\right)} \\
& =\left\|\mathcal{F}^{-1}\left(\frac{\sin (t U|\xi|)}{t U|\xi|} t U \hat{\theta}_{0}\right)\right\|_{L^{2}\left(\mathbb{R}^{3}\right)} \leq C t\left\|\theta_{0}\right\|_{L^{2}\left(\mathbb{R}^{3}\right)}
\end{aligned}
$$

Now, we estimate the term $W_{2}(t, x)=R_{2}(t, x-\cdot) *_{3} \theta_{0}$ and its derivative $\partial W_{2} / \partial t$. In order to get an $L^{2}-L^{2}$ time decay estimate for $W_{2}$ we use the Fourier transformation basing on Bochner's formula. 
The Fourier transform of an integrable, rotation-invaviant function $G(x)$ $=g(|x|)$ can be expressed by the following integral:

$$
\hat{G}(|\xi|)=\mathcal{F} G(|\xi|)=2 \pi|\xi|^{1-n / 2} \int_{0}^{\infty} g(r) r^{n / 2} J_{n / 2-1}(2 \pi|\xi| r) d r
$$

(Bochner's formula; cf. [48]). Applying it to $R_{2}$ we obtain

$$
\hat{W}_{2}(t, \xi)=\hat{R}_{2}(t, \xi) \hat{\theta}_{0}(\xi)
$$

where

$$
\hat{R}_{2}(t, \xi)=2 \pi|\xi|^{1-3 / 2} \int_{0}^{\infty} \frac{I_{1}\left(\frac{m}{U} \sqrt{U^{2} t^{2}-r^{2}}\right)}{\sqrt{U^{2} t^{2}-r^{2}}} H(U t-r) r^{3 / 2} J_{1 / 2}(2 \pi|\xi| r) d r .
$$

Taking into account the formula

$$
J_{1 / 2}(z)=\left(\frac{2}{\pi z}\right)^{1 / 2} \sin z
$$

we get

$$
\hat{R}_{2}(t, \xi)=\frac{2}{|\xi|} \int_{0}^{U t} \frac{I_{1}\left(\frac{m}{U} \sqrt{U^{2} t^{2}-r^{2}}\right)}{\sqrt{U^{2} t^{2}-r^{2}}} \sin (2 \pi|\xi| r) r d r .
$$

Changing variables according to $\sqrt{U^{2} t^{2}-r^{2}}=z$ we obtain

$$
\hat{R}_{2}(t, \xi)=\frac{2}{|\xi|} \int_{0}^{U t} I_{1}\left(\frac{m z}{U}\right) \sin \left(2 \pi|\xi| \sqrt{U^{2} t^{2}-z^{2}}\right) d z
$$

Now, we prove the following useful lemma:

LEMmA 6.3. There exists a constant $c>0$ such that for $t>0$,

$$
\left\|e^{-m t} \hat{R}_{2}(t, \xi)\right\|_{L^{\infty}\left(\mathbb{R}^{3}\right)} \leq C, \quad\left\|e^{-m t} \frac{\partial \hat{R}_{2}}{\partial t}\right\|_{L^{\infty}\left(\mathbb{R}^{3}\right)} \leq C .
$$

Proof. For given $|\xi|>0$ define

$$
f(t)=\frac{2}{|\xi|} \int_{0}^{U t} I_{1}\left(\frac{m z}{U}\right) \sin \left(2 \pi|\xi| \sqrt{U^{2} t^{2}-z^{2}}\right) d z .
$$

Then

$$
f^{\prime}(t)=4 \pi t U^{2} \int_{0}^{U t} I_{1}\left(\frac{m z}{U}\right) \frac{\cos \left(2 \pi|\xi| \sqrt{U^{2} t^{2}-z^{2}}\right)}{\sqrt{U^{2} t^{2}-z^{2}}} d z .
$$

Now, we use the following formula (cf. [46, p. 311, formula 6]):

$$
\int_{0}^{a} I_{1}(c x) \frac{\cos \left(b \sqrt{a^{2}-x^{2}}\right)}{\sqrt{a^{2}-x^{2}}} d x=\frac{1}{c a}\left[\cos \left(a \sqrt{b^{2}-c^{2}}\right)-\cos (a b)\right] .
$$


By 6.9 we obtain

$$
f^{\prime}(t)=\frac{4 U \pi}{m}\left[\cos \left(U t \sqrt{4 \pi^{2}|\xi|^{2}-m^{2} / U^{2}}\right)-\cos (U t \cdot 2 \pi|\xi|)\right] .
$$

Since $f(0)=0$, we have

$$
f(t)=\int_{0}^{t} f^{\prime}(\tau) d \tau
$$

Now, we consider two cases:

$1^{\circ}|\xi| \geq m(2 \pi U)$. In this case

$$
\left|f^{\prime}(t)\right| \leq \frac{4 U \pi}{m} \cdot 2 \leq C
$$

so we get

$$
|f(t)|=\left|\int_{0}^{t} f^{\prime}(\tau) d \tau\right| \leq C t
$$

$2^{\circ}|\xi|<m /(2 \pi U)$. Now, we obtain

$$
\begin{aligned}
& \text { 11) } \quad\left|f^{\prime}(t)\right|=\frac{4 U \pi}{m}\left|\cos \left(i t m \sqrt{1-\frac{4 \pi^{2} U^{2}|\xi|^{2}}{m^{2}}}\right)-\cos (U t \cdot 2 \pi|\xi|)\right| \\
& =\frac{4 U \pi}{m}\left|\frac{1}{2} e^{t m \sqrt{1-4 \pi^{2} U^{2}|\xi|^{2} / m^{2}}}+\frac{1}{2} e^{-t m \sqrt{1-4 \pi^{2} U^{2}|\xi|^{2} / m^{2}}}-\cos (U t \cdot 2 \pi|\xi|)\right| .
\end{aligned}
$$

Finally, from 6.11 we obtain $\left|f^{\prime}(t)\right| \leq C_{1} e^{m t}$. So we get

$$
|f(t)| \leq \int_{0}^{t}\left|f^{\prime}(\tau)\right| d \tau \leq C_{2} \int_{0}^{t} e^{m \tau} d \tau=\frac{C_{2}}{m}\left(e^{m t}-1\right) \leq C_{3} e^{m t} .
$$

In view of 6.8, $6.10,6.15$ and the definition of $f(t)$ we conclude that there exists a constant $C>0$ such that for $t>0$ and $|\xi|>0$,

$$
\left|\hat{R}_{2}(t, \xi)\right|=\left|\frac{2}{|\xi|} \int_{0}^{U t} I_{1}\left(\frac{m z}{U}\right) \sin \left(2 \pi|\xi| \sqrt{U^{2} t^{2}-z^{2}}\right) d z\right| \leq C e^{m t}
$$

and

$$
\left|\frac{\partial \hat{R}_{2}(t, \xi)}{\partial t}\right| \leq C e^{m t}
$$

The inequalities 6.12, 6.13 complete the proof of Lemma 6.3. 
Now, we will prove Theorem 6.1. Basing on Lemma 6.1 and (6.12), 6.13) we get

$$
\begin{aligned}
\left\|W_{2}\right\|_{L^{2}\left(\mathbb{R}^{3}\right)} & =\left\|\mathcal{F}^{-1}\left(\hat{R}_{2} \hat{\theta}_{0}\right)\right\|_{L^{2}\left(\mathbb{R}^{3}\right)} \leq\left\|\hat{R}_{2}\right\|_{L^{\infty}\left(\mathbb{R}^{3}\right)}\left\|\theta_{0}\right\|_{L^{2}\left(\mathbb{R}^{3}\right)} \\
& \leq C e^{m t}\left\|\theta_{0}\right\|_{L^{2}\left(\mathbb{R}^{3}\right)}, \\
\left\|\frac{\partial W_{2}}{\partial t}\right\|_{L^{2}\left(\mathbb{R}^{3}\right)} & =\left\|\mathcal{F}^{-1}\left(\frac{\partial \hat{R}_{2}}{\partial t} \hat{\theta}_{0}\right)\right\|_{L^{2}\left(\mathbb{R}^{3}\right)} \leq\left\|\frac{\partial \hat{R}_{2}}{\partial t}\right\|_{L^{\infty}\left(\mathbb{R}^{3}\right)}\left\|\theta_{0}\right\|_{2} \\
& \leq C e^{m t}\left\|\theta_{0}\right\|_{L^{2}\left(\mathbb{R}^{3}\right)} .
\end{aligned}
$$

In view of 6.4)-(6.7) and (6.14)-6.16) we have

$$
\begin{aligned}
\|\theta\|_{L^{2}\left(\mathbb{R}^{3}\right)} \leq & \left(\frac{1}{a_{0}}-\frac{\tau m}{a_{0}}\right) e^{-m t}\left\|W_{1}\right\|_{L^{2}\left(\mathbb{R}^{3}\right)}+\frac{\tau e^{-m t}}{a_{0}}\left\|\frac{\partial W_{1}}{\partial t}\right\|_{L^{2}\left(\mathbb{R}^{3}\right)} \\
& +\left(\frac{m}{4 \pi a_{0}}-\frac{m^{2} \tau}{4 \pi a_{0}}\right) e^{-m t}\left\|W_{2}\right\|_{L^{2}\left(\mathbb{R}^{3}\right)}+\frac{\tau m}{4 \pi a_{0}} e^{-m t}\left\|\frac{\partial W_{2}}{\partial t}\right\|_{L^{2}\left(\mathbb{R}^{3}\right)} \\
& \leq C\left\|\theta_{0}\right\|_{L^{2}\left(\mathbb{R}^{3}\right)} .
\end{aligned}
$$

In a similar way, we obtain

$$
\|D \theta\|_{L^{2}\left(\mathbb{R}^{3}\right)} \leq C\left\|D \theta_{0}\right\|_{L^{2}\left(\mathbb{R}^{3}\right)}
$$

7. $L^{p}-L^{q}$ time decay estimate for the solution of the Cauchy problem. Basing on Theorems 5.1, 6.1 and using interpolation methods we can now prove Main Theorem 2.1.

Proof of Main Theorem 2.1. Let $\Theta=(\theta, D \theta)$ denote the solution of the initial value problem (5.1)-(5.5) for fixed $\Theta_{0}=\left(\theta_{0}, D \theta_{0}\right) \in S^{\prime}\left(\mathbb{R}^{3}\right)$.

Now, we introduce the linear operator

$$
R: S^{\prime} \rightarrow S^{\prime}, \quad R\left(\Theta_{0}\right)=\Theta,
$$

where $\Theta_{0}=\left(\theta_{0}, D \theta_{0}\right), \Theta=(\theta, D \theta)$.

In view of the already proved Theorems 5.1, 6.1 we conclude that the operator $R$ is bounded in the spaces $W^{3,1}\left(\mathbb{R}^{3}\right), L^{2}\left(\mathbb{R}^{3}\right)$ and has the norms $\|R\|_{0},\|R\|_{1}$ in these spaces. This means that

$$
\exists C_{0}>0 \forall t>0, \quad R: W^{3,1}\left(\mathbb{R}^{3}\right) \rightarrow L^{\infty}\left(\mathbb{R}^{3}\right), \quad\|R\|_{0}=C_{0}(1+t)^{-3 / 2}
$$

and

$$
\exists C_{1}>0 \forall t>0, \quad R: L^{2}\left(\mathbb{R}^{3}\right) \rightarrow L^{2}\left(\mathbb{R}^{3}\right), \quad\|R\|_{1}=C_{1} .
$$

Taking into account the properties of Sobolev, Besov and Bessel spaces (cf. [51]), we get

$$
L^{2}\left(\mathbb{R}^{3}\right)=B_{2,2}^{0}\left(\mathbb{R}^{3}\right), \quad B_{1,1}^{3}\left(\mathbb{R}^{3}\right) \hookrightarrow W^{3,1}\left(\mathbb{R}^{3}\right) .
$$

Using interpolation between $L^{2}\left(\mathbb{R}^{3}\right)$ and $L^{\infty}\left(\mathbb{R}^{3}\right)$ we obtain

$$
\left[L^{\infty}\left(\mathbb{R}^{3}\right), L^{2}\left(\mathbb{R}^{3}\right)\right]_{2 / q}=L^{q}\left(\mathbb{R}^{3}\right) .
$$


In view of Theorem 3.4 we have

$$
\left[B_{1,1}^{3}\left(\mathbb{R}^{3}\right), B_{2,2}^{0}\left(\mathbb{R}^{3}\right)\right]_{2 / q}=B_{p, p}^{3(1-2 / q)}\left(\mathbb{R}^{3}\right) .
$$

So, basing on the interpolation theorem we conclude from $7.1,7.2$ that

$$
R: B_{p, p}^{3(1-2 / q)}\left(\mathbb{R}^{3}\right) \rightarrow L^{q}\left(\mathbb{R}^{3}\right) .
$$

Furthermore the norm of $R$ in these spaces is estimated as follows:

$$
\|R\| \leq C(1+t)^{-\frac{3}{2}(1-2 / q)} .
$$

Now, let $s \in \mathbb{N}, s \geq s_{0}$, where

$$
s_{0}=[3(1-2 / q)]+1, \quad \varepsilon:=s_{0}-3(1-2 / q) .
$$

Applying imbedding theorems and the properties of Sobolev spaces we get

$$
\begin{aligned}
W^{s, p}\left(\mathbb{R}^{3}\right) \hookrightarrow W^{s_{0}, p}\left(\mathbb{R}^{3}\right) & =H^{s_{0}, p}\left(\mathbb{R}^{3}\right) \\
& =H^{3(1-2 / q)+\varepsilon, p}\left(\mathbb{R}^{3}\right) \hookrightarrow B_{p, p}^{3(1-2 / q)}\left(\mathbb{R}^{3}\right) .
\end{aligned}
$$

Using 7.3 -7.5) we have

$$
R: W^{s, p}\left(\mathbb{R}^{3}\right) \rightarrow L^{q}\left(\mathbb{R}^{3}\right), \quad\|R\| \leq C(1+t)^{-\frac{3}{2}(1-2 / q)} .
$$

This ends the proof of Main Theorem 2.1.

Remark 7.1. Basing on Theorem 2.1 we can find an $L^{p}-L^{q}$ time decay estimate for the internal energy $e$ and heat flux $q$ (cf. formulae 2.15) and (2.16); see [20]).

8. Mathematical and physical interpretation of the solution to the nonlocal model of heat propagation with finite speed. In this section we would like to answer the question where and when the solution of the nonlocal model of heat propagation with finite speed given by formula (5.6) tends to or is equivalent to the solution of the classical heat equation.

We consider the following cases:

I: the relaxation time tends to zero,

II: we are far from the wave front of the propagation of the wave.

I. Let us assume that $\tau \rightarrow 0$. Since $m=\frac{1}{2}\left(1 / \tau+b_{0} / c\right)$ and $U=\sqrt{a_{0} / c \tau}$, if $\tau \rightarrow 0$ we have $m \rightarrow \infty, U \rightarrow \infty$ and

$$
D=\frac{U}{\sqrt{2 m}}=\sqrt{\frac{a_{0}}{c}} \text {. }
$$

Taking this into account and using (5.7) we get

$$
\theta(t, x)=\mathbb{E}_{3}(t, x-\cdot) *_{3} \theta_{0}(\cdot)+\tau\left(\partial_{t} \mathbb{E}_{3}(t, x-\cdot) *_{3} \theta_{0}(\cdot)\right)
$$


where

$$
\begin{aligned}
\mathbb{E}_{3}(t, x)= & \frac{1}{4 \pi a_{0} U^{2}|x|} e^{-m t} \delta(U t-|x|) \\
& +\frac{m e^{-m t}}{4 \pi a_{0}} \frac{I_{1}\left(\frac{m}{U} \sqrt{U^{2} t^{2}-|x|^{2}}\right)}{\sqrt{U^{2} t^{2}-|x|^{2}}} \cdot H\left(t-\frac{|x|}{U}\right)=E_{1}+E_{2} .
\end{aligned}
$$

Now, we analyse the function $E_{2}$ under the assumption 8.1.

We use the asymptotic behaviour of the Bessel function

$$
I_{1}(z)=\frac{e^{z}}{\sqrt{2 \pi z}}
$$

as $z \rightarrow \infty$. Taking it into account we have the following estimate as $\tau \rightarrow 0$ :

$$
\begin{aligned}
E_{2}(t, x) & =\frac{m e^{-m t}}{4 \pi a_{0}} \frac{I_{1}\left(\frac{m}{U} \sqrt{U^{2} t^{2}-|x|^{2}}\right)}{\sqrt{U^{2} t^{2}-|x|^{2}}} H(U t-|x|) \\
& \approx \frac{m e^{-m t}}{4 \pi a_{0} U t} \frac{e^{m t \sqrt{1-|x|^{2} / U^{2} t^{2}}}}{\sqrt{2 \pi t m \sqrt{1-|x|^{2} / U^{2} t^{2}}}} \frac{H(t-|x| / U)}{\sqrt{1-|x|^{2} / U^{2} t^{2}}} .
\end{aligned}
$$

In view of the fact that $|x|^{2} / U^{2} t^{2} \ll 1$, from 8.2 we obtain

$$
\begin{aligned}
E_{2}(t, x) & =\frac{m e^{-m t}}{4 \pi a_{0}} \frac{e^{m t-\frac{m|x|^{2}}{2 U^{2} t}}}{\sqrt{2 \pi} t^{3 / 2} \sqrt{m} U} H(t)=\frac{\sqrt{2 m} e^{-\frac{|x|^{2}}{4 D^{2} t}}}{(\sqrt{4 \pi t})^{3} a_{0} U} H(t) \\
& \underset{\tau \rightarrow 0}{\longrightarrow} \frac{e^{-\frac{|x|^{2}}{4 D^{2} t}}}{D(\sqrt{4 \pi t})^{3} a_{0}} .
\end{aligned}
$$

Now, we prove that $E_{1}(t, x-\cdot) *_{3} \theta_{0}(\cdot) \rightarrow 0$ as $\tau \rightarrow 0$. Using the definition of $\delta(U t-|x|)$ and changing variables according to $y=z U t$, we get

$$
\begin{aligned}
E_{1}(t, x-\cdot) *_{3} \theta_{0}(\cdot) & =\frac{e^{-m t}}{4 \pi a_{0}} \frac{1}{U^{3} t} \int_{|y|=U t} \theta_{0}(x-y) d S_{y} \\
& =\frac{e^{-m t} t}{4 \pi a_{0} U} \int_{|z|=1} \theta_{0}(x-U t z) d S_{z} \underset{\tau \rightarrow 0}{\longrightarrow} 0 .
\end{aligned}
$$

The derivative $\partial_{t}\left(E_{1}(t, x-\cdot) *_{3} \theta_{0}(\cdot)\right)$ can be expressed as follows:

$$
\begin{aligned}
\partial_{t}\left(E_{1}(t, x-\cdot) *_{3} \theta_{0}(\cdot)\right) & =\frac{e^{-m t} m t}{4 \pi a_{0} U} \int_{|z|=1} \theta_{0}(x-U t z) d S_{z} \\
+\frac{e^{-m t}}{4 \pi a_{0} U} \int_{|z|=1} \theta_{0}(x-U t z) d S_{z} & +\frac{e^{-m t} t}{4 \pi a_{0}} \int_{|z|=1} \nabla \theta_{0}(x-U t z) z d S_{z} \\
& =P_{1}(t, x)+P_{2}(t, x)+P_{3}(t, x) .
\end{aligned}
$$


Then, for every fixed $t>0$ we have

$$
\begin{aligned}
& \left|P_{1}\right| \leq\left\|\theta_{0}\right\|_{L^{\infty}\left(\mathbb{R}^{3}\right)} \frac{e^{-m t} m t}{4 \pi a_{0} U} \underset{\tau \rightarrow 0}{\longrightarrow} 0, \\
& \left|P_{2}\right| \leq \frac{e^{-m t}}{4 \pi a_{0} U}\left\|\theta_{0}\right\|_{L^{\infty}\left(\mathbb{R}^{3}\right)} \underset{\tau \rightarrow 0}{\longrightarrow} 0, \\
& \left|P_{3}\right| \leq \frac{e^{-m t} t}{a_{0} 4 \pi}\left\|\theta_{0}\right\|_{W^{1, \infty}\left(\mathbb{R}^{3}\right)} \underset{\tau \rightarrow 0}{\longrightarrow} 0 .
\end{aligned}
$$

The derivative $\partial_{t} E_{2}(t, x)$ can be expressed in the following form:

$$
\begin{aligned}
\partial_{t} E_{2}(t, x)= & -\tau \frac{m^{2}}{4 \pi a_{0}} e^{-m t} \frac{I_{1}\left(\frac{m}{U} \sqrt{U^{2} t^{2}-|x|^{2}}\right)}{\sqrt{U^{2} t^{2}-|x|^{2}}} H\left(t-\frac{|x|}{U}\right) \\
& +m e^{-m t} \tau\left(\frac{I_{1}^{\prime}\left(\frac{m}{U} \sqrt{U^{2} t^{2}-|x|^{2}}\right) m U t}{4 \pi a_{0}\left(U^{2} t^{2}-|x|^{2}\right)}\right. \\
& \left.-\frac{I_{1}\left(\frac{m}{U} \sqrt{U^{2} t^{2}-|x|^{2}}\right) U^{2} t / \sqrt{U^{2} t^{2}-|x|^{2}}}{4 \pi a_{0}\left(U^{2} t^{2}-|x|^{2}\right)}\right) H\left(t-\frac{|x|}{U}\right) \\
& +\tau \frac{m e^{-m t}}{4 \pi a_{0}} \frac{I_{1}\left(\frac{m}{U} \sqrt{U^{2} t^{2}-|x|^{2}}\right)}{\sqrt{U^{2} t^{2}-|x|^{2}}} \delta\left(t-\frac{|x|}{U}\right) \\
= & R_{1}(t, x)+R_{2}(t, x)+R_{3}(t, x) .
\end{aligned}
$$

For $\tau \rightarrow 0$, we have

$$
\begin{aligned}
R_{1}(t, x)= & \frac{\tau m^{2} e^{-m t}}{4 \pi a_{0}} \frac{I_{1}\left(\frac{m}{U} \sqrt{U^{2} t^{2}-|x|^{2}}\right)}{\sqrt{U^{2} t^{2}-|x|^{2}}} H\left(t-\frac{|x|}{U}\right) \\
\approx & \tau \frac{m^{2} e^{-m t}}{4 \pi a_{0} U t} \frac{e^{m t \sqrt{1-|x|^{2} / U^{2} t^{2}}}}{\sqrt{1-|x|^{2} / U^{2} t^{2}}} \frac{H(t-|x| / U)}{\sqrt{2 \pi m t \sqrt{1-|x|^{2} / U^{2} t^{2}}}} \\
\approx & \tau m \frac{\sqrt{2 m}}{a_{0} U} \frac{e^{-\frac{|x|^{2}}{4 D^{2} t}}}{(4 \pi t)^{3 / 2}} H(t) \approx \frac{1}{2 a_{0} D} \frac{e^{-\frac{|x|^{2}}{4 D^{2} t}}}{(4 \pi t)^{3 / 2}} H(t), \\
R_{2}(t, x)= & \frac{I_{1}^{\prime}\left(\frac{m}{U} \sqrt{U^{2} t^{2}-|x|^{2}}\right) \sqrt{U^{2} t^{2}-|x|^{2}} m U t-I_{1}\left(\frac{m}{U} \sqrt{U^{2} t^{2}-|x|^{2}}\right) U^{2} t}{\left(U^{2} t^{2}-|x|^{2}\right)^{3 / 2}} \\
& \cdot \frac{m e^{-m t}}{4 \pi a_{0}} \tau H(t)=\frac{\frac{1}{2} I_{0}\left(\frac{m}{U} \sqrt{U^{2} t^{2}-|x|^{2}}\right)+\frac{1}{2} I_{1}\left(\frac{m}{U} \sqrt{U^{2} t^{2}-|x|^{2}}\right)}{U^{2} t^{2}\left(1-|x|^{2} / U^{2} t^{2}\right)} \\
& \cdot \frac{m^{2} U t \cdot m e^{-m t}}{4 \pi a_{0}} \tau(t)-\frac{I_{1}\left(\frac{m}{U} \sqrt{U^{2} t^{2}-|x|^{2}}\right) U^{2} t}{U^{3} t^{3}\left(1-|x|^{2} / U^{2} t^{2}\right)^{3 / 2}} \frac{m t}{4 \pi a_{0}} \tau H(t) \\
= & R_{21}(t, x)+R_{22}(t, x)+R_{23}(t, x)
\end{aligned}
$$


where

$$
\begin{aligned}
R_{21}(t, x) & =\frac{1}{8 \pi a_{0}} m^{2} U t e^{-m t} \tau \frac{I_{0}\left(\frac{m}{U} \sqrt{U^{2} t^{2}-|x|^{2}}\right)}{U^{2} t^{2}-|x|^{2}} H(t) \\
& \approx \frac{1}{8 \pi a_{0} t^{3 / 2}} \frac{1}{\sqrt{2 \pi}} \frac{m^{3 / 2} \tau}{U} e^{-\frac{|x|^{2} m}{U^{2} t^{2}}} \approx \frac{1}{2 a_{0} D(4 \pi t)^{3 / 2}} e^{-\frac{|x|^{2}}{4 D^{2} t} H(t),} \\
R_{22}(t, x) & =\frac{t}{8 \pi a_{0}} m^{2} U e^{-m t} \tau \frac{I_{1}\left(\frac{m}{U} \sqrt{U^{2} t^{2}-|x|^{2}}\right)}{U^{2} t^{2}-|x|^{2}} \\
& \approx \frac{1}{2 a_{0} D(4 \pi t)^{3 / 2}} e^{-\frac{|x|^{2}}{4 D^{2} t} H(t),} \\
R_{23}(t, x) & =\frac{-1}{4 \pi a_{0}} \tau m e^{-m t} \frac{U^{2} t}{U^{3} t^{3}\left(1-|x|^{2} / U^{2} t^{2}\right)^{3 / 2}} I_{1}\left(\frac{m}{U} \sqrt{U^{2} t^{2}-|x|^{2}}\right) \\
& \approx \frac{-1}{4 \pi a} \frac{1}{t^{2}} e^{-m t} \frac{m \tau}{U} \frac{e^{m t \sqrt{1-|x|^{2} / U^{2} t^{2}}}}{\sqrt{2 \pi m t \sqrt{1-|x|^{2} / U^{2} t^{2}}}} \approx 0 .
\end{aligned}
$$

Now, we consider the term $R_{3}(t, x)$ :

$$
\begin{aligned}
R_{3}(t, x) & =\frac{\tau m e^{-m t}}{4 \pi a_{0}} \frac{m}{U} \frac{I_{1}\left(\frac{m}{U} \sqrt{U^{2} t^{2}-|x|^{2}}\right)}{\frac{m}{U} \sqrt{U^{2} t^{2}-|x|^{2}}} \delta\left(t-\frac{|x|}{U}\right) \\
& =\frac{\tau m e^{-m t}}{4 \pi a_{0}} \frac{m}{U} \frac{1}{2} \delta\left(t-\frac{|x|}{U}\right) .
\end{aligned}
$$

Here we use the fact that $\left.\frac{I_{1}(z)}{z}\right|_{z=0}=\frac{1}{2}$. From 8.3 and the definition of Dirac distribution we get

$$
R_{3}(t, x) * \theta_{0} \underset{\tau \rightarrow 0}{\longrightarrow} 0 .
$$

Taking into account all the above considerations we have

$$
\begin{aligned}
\theta(t, x)= & E_{1}(t, x-\cdot) *_{3} \theta_{0}(\cdot)+E_{2}(t, x-\cdot) *_{3} \theta_{0}(\cdot) \\
& +\tau\left(\partial_{t} E_{1}(t, x-\cdot) *_{3} \theta_{0}(\cdot)\right)+\tau\left(\partial_{t} E_{2}(t, x-\cdot) *_{3} \theta_{0}(\cdot)\right) \\
\approx & \frac{3 e^{-\frac{|x|^{2}}{4 D^{2} t}}}{2 a_{0} D(4 \pi t)^{3 / 2}} *_{3} \theta_{0}(\cdot) .
\end{aligned}
$$

The last formula proves that if $\tau \rightarrow 0$, then the solution $\theta$ of the Cauchy problem for the nonlocal model of heat propagation is equivalent to the solution of the Cauchy problem for the classical parabolic heat equation.

Now, we consider the second case:

II. If

$$
\frac{|x|}{U t} \ll 1
$$


(while $\tau$ is fixed), it means that we are very far from the wave front of propagation for a very long time. For the finite value $U$ of wave speed, putting $m=U^{2}\left(c+b_{0} \tau\right) / a_{0}$ in (5.6), after some calculation we get

$$
\mathbb{E}(t, x)=\mathbb{E}_{2}(t, x)=\frac{e^{\frac{-|x|^{2}}{4 D_{0}^{2} t}}}{D_{0}(\sqrt{4 \pi t})^{3} a_{0}} \quad\left(\mathbb{E}_{1}=0\right)
$$

where now $D_{0}^{2}=\frac{a_{0}}{c+b_{0} \tau}=D^{2} \frac{1}{1+b_{0} \tau / c} \approx D^{2}$ (in view of the small value of the relaxation time $\tau$ ).

We can give the following interpretation of these facts:

The solution under the nonlocal model is equivalent to the classical solution if:

I. $U=\sqrt{a_{0} / \tau c} \gg 1$ (which means that $\tau \rightarrow 0$ ) (cf. Fig. 4);

II. $|x| / U t \ll 1$ for $\tau>0$ (which means that we are very far from the front of the thermal wave) (cf. Fig. 5).

Conclusion. The nonlocal model of heat propagation with finite speed is essentially different from the classical description of heat propagation for the following reasons:

- the wave front exists in this description (cf. Fig. 5);

- the distribution of the temperature exists in a neighbourhood of the wave front (cf. Fig. 5).

9. Numerical calculations. In this section we will calculate the distribution of the temperature assuming that the source is given by the formula

$$
W(t, x)=W_{0} f(t) \delta\left(x_{1}\right) \delta\left(x_{2}\right) H\left(l-\left|x_{3}\right|\right) .
$$

Applying the Laplace transformation with respect to $t$ and Fourier transformation with respect to $x_{1}, x_{2}$ to equation 4.1) (putting $E(t, x) \equiv \theta(t, x)$ ) with $W(t, x)$ given by 9.1 we get

$$
-\frac{d^{2} \hat{\bar{\theta}}}{d x_{3}^{2}}+\gamma^{2} \hat{\bar{\theta}}=\frac{1}{\bar{a}} W_{0} F(s) H\left(l-\left|x_{3}\right|\right) .
$$

In view of 4.2 the solution of 9.2 is given as follows:

$$
\hat{\bar{\theta}}\left(s, \xi_{1}, \xi_{2}, x_{3}\right)=\frac{W_{0}}{2 \bar{a} \gamma} F(s)\left(e^{-\gamma x_{3}} * H\left(l-\left|x_{3}\right|\right)\right),
$$

that is,

$$
\hat{\bar{\theta}}\left(s, \xi_{1}, \xi_{2}, x_{3}\right)=\frac{W_{0}}{2 \bar{a} \gamma} F(s) \int_{-l}^{l} e^{-\gamma\left|x_{3}-\xi\right|} d \xi,
$$


so we get

$$
\begin{aligned}
\hat{\bar{\theta}}\left(s, \xi_{1}, \xi_{2}, x_{3}\right)=\frac{W_{0}}{2 \bar{a}} F(s)\left(\frac { 1 } { \gamma ^ { 2 } } \left(2 H\left(l-\left|x_{3}\right|\right)\right.\right. & -\operatorname{sgn}\left(x_{3}+l\right) \cdot e^{-\gamma\left|x_{3}+l\right|} \\
& \left.\left.+\operatorname{sgn}\left(x_{3}-l\right) e^{-\gamma\left|x_{3}-l\right|}\right)\right) .
\end{aligned}
$$

The last formula can be written in the form

$$
\begin{aligned}
\hat{\bar{\theta}}\left(s, \xi_{1}, \xi_{2}, x_{3}\right)= & \frac{W_{0} F(s)}{2 \bar{a}(s)}\left(\frac { 1 } { \gamma ^ { 2 } } \left(\lim _{\varepsilon \rightarrow 0} 2 H\left(l-\left|x_{3}\right|\right) e^{-\gamma \varepsilon}\right.\right. \\
& \left.\left.-\operatorname{sgn}\left(x_{3}+l\right) e^{-\gamma\left|x_{3}+l\right|}+\operatorname{sgn}\left(x_{3}-l\right) e^{-\gamma\left|x_{3}-l\right|}\right)\right) .
\end{aligned}
$$

Using 4.3-4.7 from 9.3 we get

(9.4) $\bar{\theta}\left(s, \varrho, x_{3}\right)$

$$
=\left.\frac{W_{0} F(s)}{8 \pi^{2} \bar{a}} \int_{-\infty}^{\infty} d q \sum_{k=0}^{2} c_{k} \int_{R_{k} \sqrt{q^{2}+1}}^{\infty} \frac{\bar{r} e^{-\bar{r} T}}{\left(\sqrt{\omega_{k}^{2}+q^{2}+1}\right)^{2}} \frac{\partial \omega_{k}}{\partial T}\right|_{\omega_{k}=\omega_{k}^{-}} ^{\omega_{k}=\omega_{k}^{+}} d T
$$

where

$$
\begin{aligned}
& c_{0}=\lim _{\varepsilon \rightarrow 0} 2 H\left(l-\left|x_{3}\right|\right) e^{-\gamma \varepsilon}, \quad c_{1}=-\operatorname{sgn}\left(x_{3}+l\right), \quad c_{2}=\operatorname{sgn}\left(x_{3}-l\right), \\
& R_{0}=\sqrt{\varrho^{2}+\varepsilon^{2}}, \quad R_{1}=\sqrt{\varrho^{2}+\left(x_{3}+l\right)^{2}}, \quad R_{2}=\sqrt{\varrho^{2}+\left(x_{3}-l\right)^{2}},
\end{aligned}
$$

and

$$
\begin{aligned}
& \omega_{k}^{ \pm}=i \frac{T \varrho}{R_{k}^{2}} \pm \frac{\left|x_{3}\right| k}{R_{k}^{2}} \sqrt{T^{2}-R_{k}^{2}\left(q^{2}+1\right)}, \quad \varrho^{2}=x_{1}^{2}+x_{2}^{2}, \\
& \gamma_{k}^{ \pm}=T \frac{\left|x_{3}\right|_{k}}{R_{k}^{2}} \pm i \frac{\varrho}{R_{k}^{2}} \sqrt{T^{2}-R_{k}^{2}\left(q^{2}+1\right)} \\
& \frac{\partial \omega_{k}^{ \pm}}{\partial T}= \pm \frac{\gamma_{k}^{ \pm}}{\sqrt{T^{2}-R_{k}^{2}\left(q^{2}+1\right)}}
\end{aligned}
$$

and $\left|x_{3}\right|_{0}=|\varepsilon|,\left|x_{3}\right|_{1}=\left|x_{3}+l\right|,\left|x_{3}\right|_{2}=\left|x_{3}-l\right|$.

Taking into account (9.3), 9.5) we can write (9.4) as follows:

$$
\bar{\theta}\left(s, \varrho, x_{3}\right)=\frac{W_{0} F(s)}{4 \pi^{2} \bar{a}(s)} \int_{-\infty}^{\infty} d q \sum_{k=0}^{2} c_{k} \int_{R_{k} \sqrt{q^{2}+1}}^{\infty} \frac{\bar{r}\left|x_{3}\right|_{k} T e^{-\bar{r} T} d T}{\sqrt{T^{2}-R_{k}^{2}\left(q^{2}+1\right)}\left(T^{2}-\varrho^{2}\left(q^{2}+1\right)\right)}
$$


and after some calculations

$$
\begin{aligned}
\bar{\theta}\left(s, \varrho, x_{3}\right)= & \frac{W_{0} F(s)}{4 \pi^{2} \bar{a}(s)} \sum_{k=0}^{2} c_{k} \int_{R_{k}}^{\infty} \bar{r}\left|x_{3}\right|_{k} T e^{-\bar{r} T} d T \\
& \quad \int_{\left(T / R_{k}\right)^{2}-1} \frac{d q}{\sqrt{\tau^{2}-R_{k}^{2}\left(q^{2}+1\right)}\left(T^{2}-\varrho^{2}\left(q^{2}+1\right)\right)}
\end{aligned}
$$

where $\left(T / R_{k}\right)^{2}-1>0$. The last formula can be written as follows:

$$
\bar{\theta}\left(s, \varrho, x_{3}\right)=\frac{W_{0} F(s)}{4 \pi^{2} \bar{a}(s)} \sum_{k=0}^{2} c_{k} \int_{R_{k}}^{\infty}\left(\frac{\bar{r}\left|x_{3}\right|_{k} T e^{-\bar{r} T}}{R_{k} \cdot \varrho^{2}} \int_{-1}^{1} \frac{d u}{\sqrt{1-u^{2}}\left(a^{2}-b^{2} u^{2}\right)}\right) d T
$$

where $a^{2}=(T / \varrho)^{2}-1, b^{2}=\left(T / R_{k}\right)^{2}-1$. After some calculations we get

$$
\bar{\theta}\left(s, \varrho, x_{3}\right)=\frac{W_{0} F(s)}{4 \pi^{2} \bar{a}(s)} \sum_{k=0}^{2} c_{k} \int_{R_{k}}^{\infty} \frac{\bar{r}\left|x_{3}\right|_{k} T e^{-\bar{r} T}}{R_{k} \varrho^{2}} \cdot \frac{R_{k} \varrho(-\pi)}{T\left|x_{3}\right|_{k} \sqrt{T^{2} / \varrho^{2}-1}} d T
$$

and finally

$$
\bar{\theta}\left(s, \varrho, x_{3}\right)=\frac{W_{0} F(s)}{4 \pi \bar{a}(s)} \sum_{k=0}^{2}\left(-c_{k}\right) \int_{R_{k}}^{\infty} \frac{\bar{r} e^{-\bar{r} T}}{\sqrt{T^{2}-\varrho^{2}}} d T .
$$

Applying to (9.6) the inverse Laplace transform, we get

$$
=\theta_{0} \sum_{k=0}^{2}\left(-c_{k}\right) H\left(\frac{t}{t_{0}}-\frac{R_{k}}{U t_{0}}\right) e^{-\frac{t}{t_{0}}} \cdot \int_{R_{k}}^{U t} \frac{I_{0} \sqrt{\left(\frac{t}{t_{0}}\right)^{2}-\left(\frac{T}{U t_{0}}\right)^{2}}}{\sqrt{T^{2}-\varrho^{2}}} d T
$$

where

$$
R_{0}=\varrho=\sqrt{x_{1}^{2}+x_{2}^{2}}, \quad R_{1}=\sqrt{\varrho^{2}+\left(x_{3}+l\right)^{2}}, \quad R_{2}=\sqrt{\varrho^{2}+\left(x_{3}-l\right)^{2}} .
$$

Taking into account (9.7) we performed the numerical calculations which are presented in Figures 24 .

The numerical analysis of the solutions leads to the conclusion that there are three waves propagating in space-time. The first of them is the cylindrical wave described by the equations

$$
\varrho-v t=0, \quad \varrho=\sqrt{x_{1}^{2}+x_{2}^{2}} \quad \text { for } x_{3} \in[-1,1] .
$$




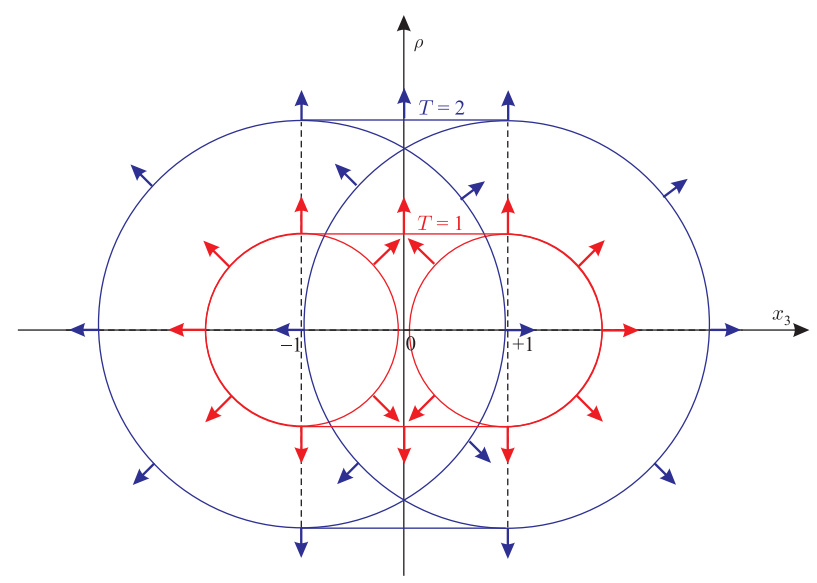

Fig. 2. Space distribution of the wave surface for $T_{1}=1, T_{2}=2$ ( $T$ is nondimensional time)

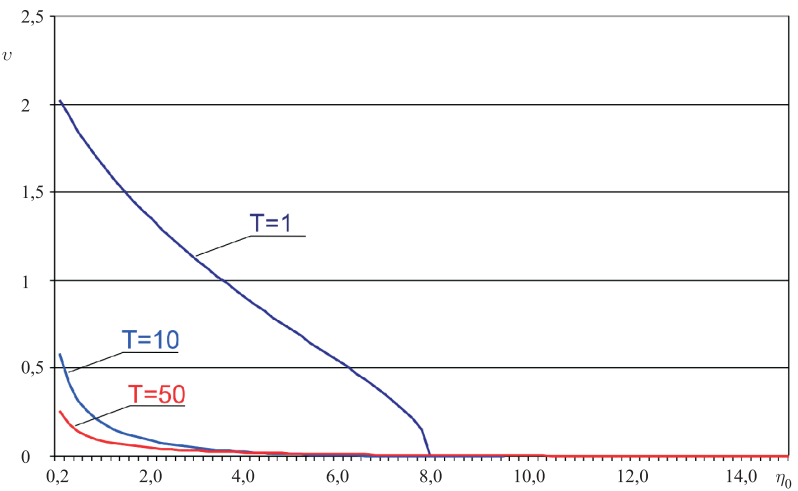

Fig. 3. Distribution of the temperature for the nonlocal model of heat propagation $(x=0$, $T=1,10,50)$

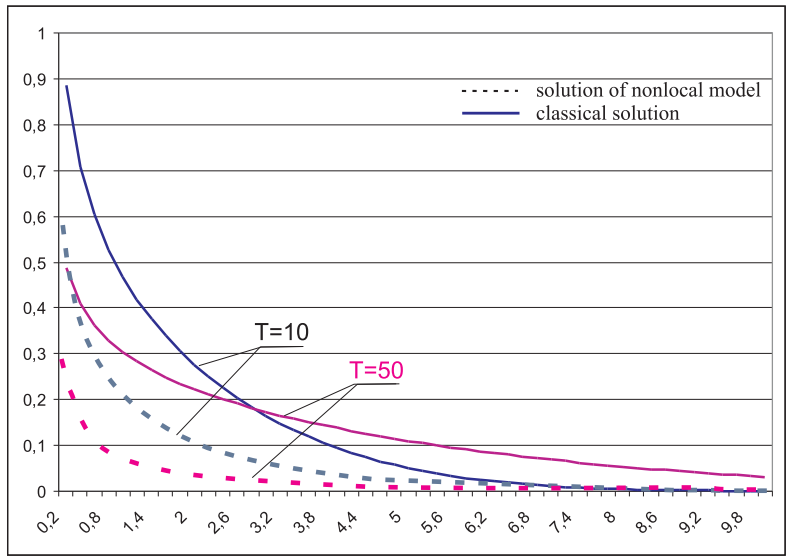

Fig. 4. Distribution of the nondimensional temperature as a function of distance from the source $\left(x_{3}=0, T=10, T=50\right)$ 
Next, there are two spherical waves tangent to the surface of the cylinder given by the equations

$$
R_{k}-U t=0, \quad R_{1}=\sqrt{\varrho^{2}+\left(x_{3}+1\right)^{2}}, \quad R_{2}=\sqrt{\varrho^{2}+\left(x_{3}-1\right)^{2}} .
$$

This means that there are two spheres with centres at $S_{1}(0,0,-1)$ and $S_{2}(0,0,1)$ and of radius $U t$.

We can see the propagation of the two waves in Figure 2 for $T_{1}=U t_{1}=1$ and $T_{2}=U t_{2}=2$.

The distribution of the temperature is presented in Figure 3 for $T=$ $1,10,50$.

In order to compare the solution of the Gurtin-Pipkin model of heat propagation with finite speed with the classical Fourier model with infinite heat propagation speed we performed the numerical calculations basing on formula (9.7). The results are presented in Figures 4 and 5 , respectively.

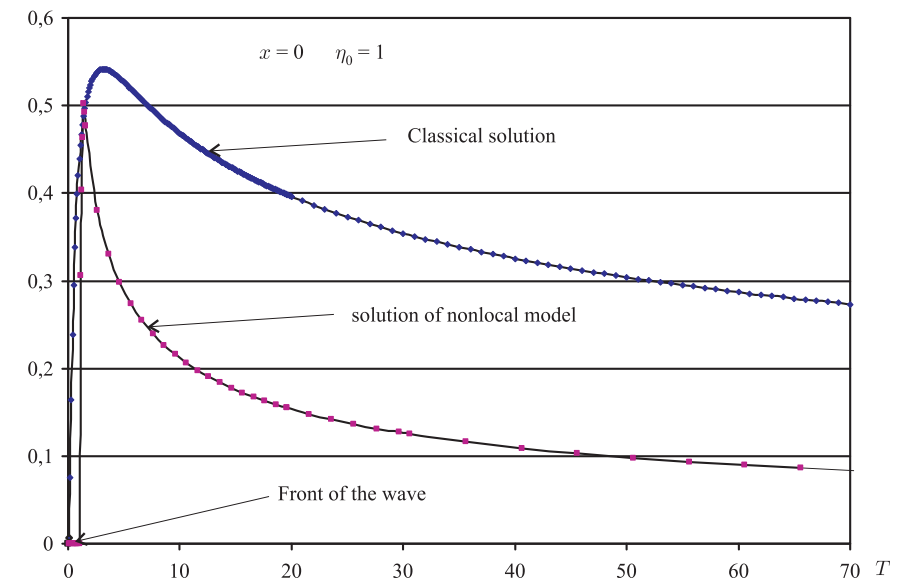

Fig. 5. Distribution of the temperature as a function of time (for $x_{3}=0, \varrho=1$ ). In the graph we see that in the solution of the nonlocal model the front of the initial wave appears $(T=1)$. The temperature before the wave front is zero $(\theta=0)$ for $0 \leq T \leq 1$. In the case of the classical solution the temperature is greater than zero for all $T>0$.

These results confirm the theoretical considerations of Section 8 .

REMARK 9.1. It is worth mentioning that the $L^{p}-L^{q}$ time decay estimate for the solution of the Cauchy problem plays the main role in the proof of the global existence for the Cauchy problem for the nonlinear nonlocal model associated with the linear one considered in this paper.

Acknowledgements. The authors thank Dr. M. Pasternak from the Department of Electronics, Military University of Technology, Warsaw, for his help in the numerical calculations. 


\section{References}

[1] C. C. Ackerman, B. Bentman, H. A. Fairbank and R. A. Guyer, Second sound in solid helium, Phys. Rev. Lett. 16 (1966), 789-791.

[2] C. C. Ackerman and W. C. Overton, Second sound in solid helium - 3, Phys. Rev. Lett. 22 (1969), 764-766.

[3] R. A. Adams, Sobolev Spaces, Academic Press, New York, 1975.

[4] K. R. Atkins and D. V. Osborne, The velocity of second sound below $1^{\circ} \mathrm{K}$, Philos. Mag. 41 (1950), 1078-1081.

[5] E. F. Beckenbach, Modern Mathematics for the Engineer, McGraw-Hill, New York, 1961.

[6] B. Bertman and R. J. Sandiford, Second sound in solid helium, Sci. Amer. 222 (1970), no. 5, 92-101.

[7] L. Cagniard, Reflection and Refraction of Progressive Seismic Waves, McGraw-Hill, New York, 1962.

[8] C. Cattaneo, On the conduction of heat, Atti Sem. Mat. Fis. Univ. Modena 3 (1948), $3-21$.

[9] C. Cattaneo, A form of heat equation which eliminates the paradox of instantaneous propagation, C. R. Acad. Sci. Paris 247 (1958), 431-433.

[10] D. S. Chandrasekharaiah, Thermoelasticity with second sound: A review, Appl. Mech. Rev. 39 (1986), 355-376.

[11] M. Chester, Second sound in solids, Phys. Rev. 131 (1963), 2013-2015.

[12] B. D. Coleman and W. Noll, An aproximation theorem for functionals, with applications in continuum mechanics, Arch. Ration. Mech. Anal. 6 (1960), 355-370.

[13] R. B. Dingle, Remarks on two-liquid model of Helium II, Philos. Mag. 42 (1951), 1080-1088.

[14] R. B. Dingle, Derivation of the velocity of second sound from Maxwell's equation of transfer, Proc. Phys. Soc. 1952, A65, 364-376.

[15] G. D. Fiodor, Laplace Transforms in Engineering, Akadémiai Kiadó, Budapest, 1965.

[16] P. H. Francis, Thermo-mechanical effects in elastic wave propagation. A survey, J. Sound Vib. 21 (1972), 181-192.

[17] J. Gawinecki, Global solution to the Cauchy problem in non-linear hyperbolic thermoelasticity, Math. Methods Appl. Sci. 15 (1992), 223-237.

[18] J. Gawinecki, Global solutions to initial value problems in nonlinear hyperbolic thermoelasticity, Dissertationes Math. 344 (1995), 61 pp.

[19] J. Gawinecki and Do Duc Hung, Global existence of solution to the initial value problem for nonlinear hyperbolic heat equation, Bull. Polish Acad. Sci. Math. 39 (1991), 21-29.

[20] J. Gawinecki, J. Rafa and E. Włodarczyk, $L^{\infty}-L^{1}$-time decay estimate to the solution of the Cauchy problem of the system of equations describing nonlocal model of the propagation of heat with finite speed, Biul. WAT 42 (1993), no. 12, 4-20.

[21] I. S. Gradshteĭn and I. I. Ryzhik, Tables of Integrals, Sums, Series and Products, Nauka, 1971 (in Russian).

[22] M. E. Gurtin and A. C. Pipkin, A general theory of heat conduction with finite wave speeds, Arch. Ration. Mech. Anal. 31 (1968), 113-126.

[23] R. J. von Gutfeld and A. H. Nethercot, Temperature dependence of heat-pulse propagation in sapphire, Phys. Rev. Lett. 17 (1966), 868-871.

[24] A. T. de Hoop, A modification of Cagniard's methods of solving seismic pulse problems, Appl. Sci. Res. Sec. B 8 (1959), 349-356. 
[25] L. Hörmander, The Analysis of Linear Partial Differential Operators, Vol. 2, Differential Operators with Constant Coefficients, Springer, Berlin, 1989.

[26] L. Hörmander, Estimates for translation invariant operators in $L^{p}$ spaces, Acta Math. 104 (1960), 93-140.

[27] H. E. Jackson and C. T. Walker, Thermal conductivity, second sound and phononphonon interactions in NaF, Phys. Rev. B 3 (1971), 1428-1439.

[28] H. E. Jackson, C. T. Walker and T. W. McNelly, Second sound in NaF, Phys. Rev. Lett. 25 (1970), 26-28.

[29] S. Kaliski, Wave equation of heat conduction, Bull. Acad. Polon. Sci. Sér. Sci. Tech. 13 (1965), 211-219.

[30] S. Kaliski, Wave equations of thermoelasticity, Bull. Acad. Polon. Sci. Sér. Sci. Tech. 13 (1965), 253-260.

[31] J. Lambermont and G. Lebon, On a generalization of the Gibbs equation for heat conduction, Phys. Lett. A 42 (1973), 499-500.

[32] L. D. Landau, The theory of superfluidity of helium II, J. Phys. USSR 5 (1941), 71-90.

[33] E. M. Lifshitz, Superfluidity, Sci. Amer. 198 (1958), no. 6, 30-36.

[34] F. London, Superfluids, Wiley, Now York, 1954, Vol. II, p. 101.

[35] A. V. Luikov, Application of methods of thermodynamics of irreversible process to investigation of heat and mass exchange, J. Engrg. Phys. 9 (1965), 287-304.

[36] A. V. Luikov, Application of irreversible thermodynamics methods to investigation of heat and mass transfer, Int. J. Heat Mass Transfer (1966), no. 9, 139-152.

[37] M. J. Maurer, Relaxation model for heat conduction in metals, J. Appl. Phys. 40 (1969), 5123-5130.

[38] R. D. Maurer and M. A. Herlin, Second sound velocity in helium II, Phys. Rev. 76 (1949), 948-950.

[39] J. C. Maxwell, On the dynamic theory of gases, Philos. Trans. Roy. Soc. London 157 (1867), 49-88.

[40] T. F. McNelly, S. J. Rogers, D. J. Channin, R. J. Rollefson, W. M. Goubau, G. E. Schmidt, J. A. Krumhansl, and R. O. Pohl, Heat pulses in NaF: Onset of second sound, Phys. Rev. Lett. 24 (1970), 100-102.

[41] R. E. Nettleton, Relaxation theory of thermal conduction in liquids, Phys. Fluids 3 (1960), 216-225.

[42] W. Nowacki, Dynamic Problems of Thermoelasticity, Noordhoff, Leyden.

[43] J. W. Nunziato, On heat conduction in materials with memory, Quart. Appl. Math. 29 (1971), 187-204.

[44] J. R. Pellam and R. B. Scott, Second sound velocity in paramagnetically cooled liquid helium II, Phys. Rev. 76 (1949), 869-870.

[45] V. Peshkov, Second sound in helium II, J. Phys. USSR 8 (1944), 131.

[46] A. P. Prudnikov, Yu. A. Brychkov and O. I. Marichev, Integrals and Series. Special Functions, Nauka, 1983 (in Russian).

[47] S. J. Rogers, Transport of heat and approach to second sound in some isotopically pure alkali-halide crystals, Phys. Rev. 3 (1971), 1440-1457.

[48] L. Schwartz, Théorie des distributions, nouvelle éd., Hermann, Paris, 1966.

[49] S. L. Sobolev, Applications of Functional Analysis in Mathematical Physics, Nauka, Moscow, 1988 (in Russian).

[50] L. Tisza, The theory of liquid helium, Phys. Rev. 72 (1947), 838-839.

[51] H. Triebel, Theory of Function Spaces, Birkhäuser, Basel, 1983.

[52] P. Vernotte, The true heat equation, C. R. Acad. Sci. Paris 247 (1958), 103.

[53] P. Vernotte, Paradoxes in the continuous theory of the heat equation, C. R. Acad. Sci. Paris 246 (1958), 3154-3158. 
[54] V. Vladimirov, V. Mikhailov, M. Chabounine, Kh. Karimov, Y. Sidorov et A. Vacharine, Recueil de Problèmes d'Équations de Physique Mathématique, Mir, Moscow, 1976.

[55] J. C. Ward and J. Wilks, The velocity of second sound in liquid helium near absolute zero, Philos. Mag. 42 (1951), 314-316.

[56] J. C. Ward and J. Wilks, Second sound and the thermomechanical effect at very low temperatures, Philos. Mag. 43 (1952), 48-50.

Jerzy A. Gawinecki, Jarosław Łazuka, J. Rafa

Agnieszka Gawinecka

Institute of Mathematics and Cryptology

Faculty of Cybernetics

Military University of Technology

Kaliskiego 2

Central Military Bureau

00-908 Warszawa, Poland

E-mail: jgawinecki@wat.edu.pl of Design and Technology Kaliskiego 2 00-908 Warszawa, Poland

Received on 18.5.2011;

revised version on 2.8.2011 and 17.7.2012 
